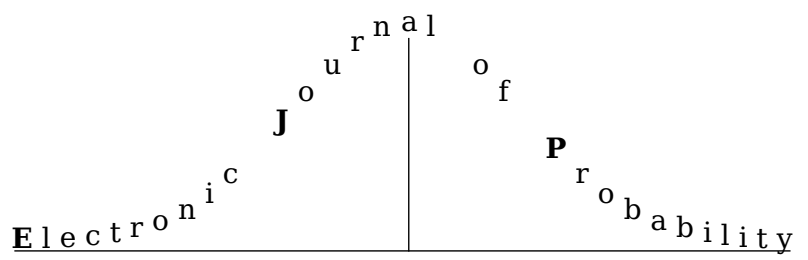

Electron. J. Probab. 25 (2020), no. 44, 1-34.

ISSN: 1083-6489 https://doi.org/10.1214/20-EJP440

\title{
Weak symmetries of stochastic differential equations driven by semimartingales with jumps*
}

\author{
Sergio Albeverio ${ }^{\dagger} \quad$ Francesco C. De Vecchi $^{\ddagger} \quad$ Paola Morando $^{\S}$ \\ Stefania Ugolini"
}

\begin{abstract}
Stochastic symmetries and related invariance properties of finite dimensional SDEs driven by general càdlàg semimartingales taking values in Lie groups are defined and investigated. The considered set of SDEs, first introduced by S. Cohen, includes affine and Marcus type SDEs as well as smooth SDEs driven by Lévy processes and iterated random maps. A natural extension to this general setting of reduction and reconstruction theory for symmetric SDEs is provided. Our theorems imply as special cases non trivial invariance results concerning a class of affine iterated random maps as well as (weak) symmetries for numerical schemes (of Euler and Milstein type) for Brownian motion driven SDEs.
\end{abstract}

Keywords: Lie symmetry analysis; stochastic differential equations; semimartingales with jumps; stochastic processes on manifolds.

AMS MSC 2010: 60H10; 60G45; 58D19.

Submitted to EJP on April 26, 2019, final version accepted on March 2, 2020.

\section{Introduction}

The study of symmetries and invariance properties of ordinary and partial differential equations (ODEs and PDEs, respectively) is a classical and well-developed research field (see $[10,51])$ and provides a powerful tool for both computing some explicit solutions to the equations and analyzing their qualitative behavior.

The study of invariance properties of finite or infinite dimensional stochastic differential equations (SDEs) is, in comparison, less developed and a systematic study could be fruitful from both the practical and the theoretical point of view.

The knowledge of some closed formulas is important in many applications of stochastic processes since it permits to develop faster and cheaper numerical algorithms for the

\footnotetext{
*The second author is supported by the German Research Foundation (DFG) via CRC 1060

${ }^{\dagger} \mathrm{HCM}$, Universität Bonn, Germany. E-mail: sergio.albeverio@iam. uni-bonn.de

${ }^{\ddagger} \mathrm{HCM}$, Universität Bonn, Germany. E-mail: francesco. devecchi@uni - bonn. de

${ }^{\S}$ DISAA, Università degli studi di Milano, Italy. E-mail: paola.morando@unimi.it

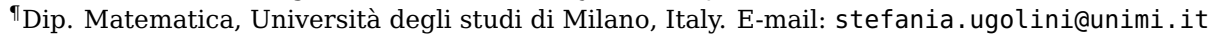


simulation of the process or to evaluate interesting quantities related to it. Moreover, the use of closed formulas allows the application of simpler statistical methods for the calibration of models (this is the reason for the popularity of affine models in mathematical finance, see e.g. [21,31], or of the Kalman filter and its generalizations in the theory of stochastic filtering, see e.g. [8]). The presence of symmetries and invariance properties is a strong clue for the possibility of closed formulas (see, for example, [18, 19], where classical infinitesimal symmetry techniques are used for finding fundamental solutions of some diffusion processes applied in mathematical finance, or [16, 17, 23], where geometrical methods based on Lie algebras are used to find new finite dimensional stochastic filters).

The investigation of invariance properties is relevant also from a theoretical point of view, in particular when stochastic processes are discussed in a geometrical framework. Some interesting examples of this approach are the study of Lévy processes on Lie groups [2, 47], the geometric description of stochastic filtering (see [33], where invariant diffusions on fiber bundles are discussed), and the study of variational stochastic systems $[20,55]$.

In this paper we apply Sophus Lie original ideas to the study of stochastic symmetries of a finite dimensional SDE driven by general càdlàg semimartingales taking values in Lie groups. In particular, we introduce a group of transformations which change both the processes solving the considered SDE and its driving noise, and transform correspondingly the coefficients of the SDE. Therefore, we look for the subgroup of all transformations which leave invariant the set of solutions of a given SDE.

In order to clarify the novelty of our study we describe, without claiming to be exhaustive, some previous results on the same problem. There are essentially two natural approaches to the description of the symmetries of a SDE. The first one, applied when the solution processes are Markovian semimartingales, consists in studying the invariance properties of the generator of the SDE solutions (which is an analytical object). This approach, used by Glover et al. [35], Cohen de Lara [15] and Liao [48], deals with a large group of transformations involving both a general spatial transformation and a solution-dependent stochastic time change. See [26] for a martingale approach in the same direction.

The second line, mainly applied to Brownian-motion-driven SDEs, consists in restricting the attention to a suitable set of transformations and directly applying a natural notion of symmetry, closely inspired by the ODEs case (see Gaeta et al. [34] for SDEs driven by Brownian motion and Lázaro-Camí and Ortega [44] for SDEs driven by general continuous semimartingales). Recently (see [27]) the family of stochastic transformations has been enriched by introducing the change of the underlying probability measure via Girsanov theorem, recovering in this way all the Lie point symmetries of the associated Kolmogorov equation. We do not investigate this topic here.

Both approaches have their strengths and weakness. The first method permits to treat a very general family of processes (all Markovian processes on a metric space) and a large class of transformations with interesting applications (see [35, 48]), but the explicit calculation of the symmetries is quite difficult in the non-diffusive case. Conversely, the second approach allows us to face the non-Markovian setting (see [44]) and permits easy explicit calculations. In particular, in this framework, it is possible to get the determining equations, that are a set of first order PDEs which uniquely characterizes the symmetries of the SDE. As the first approach, also the second one has interesting applications (see, e.g., $[44,24]$ ), even though, until now, it has been confined to the case of continuous semimartingales.

In this paper, aiming at reducing the gap between these two approaches, we propose a possible foundation of the concept of symmetry for general SDEs and we extend 
the methods introduced in [25] where, despite working in the setting of the second approach, we introduced a large family of transformations which allowed us to obtain all the symmetries of the first setting for Brownian-motion-driven SDEs.

Let us recall that in [25] we considered an SDE as a pair $(\mu(x), \sigma(x))$, where $\mu$ is the drift and $\sigma$ is the diffusion coefficient defined on a manifold $M$ and we called solution to the $\operatorname{SDE}(\mu, \sigma)$ a pair $(X, W)$, where $X$ is a semimartingale on $M$ and $W$ is an $n$ dimensional Brownian motion. A stochastic transformation, according to [25], is a triple $T=(\Phi, B, \eta)$, where $\Phi$ is a diffeomorphism of $M, B$ is a $X_{t}$-dependent rotation and $\eta$ is a $X_{t}$-dependent density of a stochastic time change. The transformation $T$ induces an action $E_{T}$ on the $\operatorname{SDE}(\mu, \sigma)$ and an action $P_{T}$ on the process $(X, W)$. The operator $P_{T}$ acts on the process $(X, W)$ changing the semimartingale $X$ by the diffeomorphism $\Phi$ and the time change $\int_{0}^{t} \eta d t$, and on the Brownian motion $W$ by the rotation $B$ and the same time change. Since the Brownian motion is invariant with respect to both rotations and time rescaling, the process $P_{T}(X, W)$ is composed by a semimartingale on $M$ and a new $n$-dimensional Brownian motion. The action $E_{T}$ of the stochastic transformation on $(\mu, \sigma)$ is the unique way of changing the $\operatorname{SDE}$ so that, if $(X, W)$ is a solution to $(\mu, \sigma)$, then $P_{T}(X, W)$ is a solution to $E_{T}(\mu, \sigma)$.

In this framework a symmetry is defined as a transformation $T$ which leaves the $\operatorname{SDE}(\mu, \sigma)$ invariant. These transformations are the only ones which preserve the set of solutions to the $\operatorname{SDE}(\mu, \sigma)$. Since all actions $P_{T}$ and $E_{T}$ are explicitly determined in terms of $T=(\Phi, B, \eta)$, it is possible to write the determining equations satisfied by $T$ which can be solved explicitly with a computer algebra software (see [24]).

The main aim of the present paper is to generalize this approach from Brownianmotion-driven SDEs to SDEs driven by general càdlàg semimartingales taking values in (finite dimensional) Lie groups. There are two main differences with respect to the Brownian motion setting. The first one is the lack of a natural geometric transformation rule for processes with jumps replacing the Itô transformation rule for continuous processes. This fact makes the action of a diffeomorphism $\Phi$ on a SDE with such general driving noise more difficult to be described. The second one is the fact that a general semimartingale does not have the symmetry properties of Brownian motion in the sense that we cannot "rotate" it or make general time changes.

In order to address the first problem we restrict our study to a particular family of SDEs (that we call geometrical SDEs) introduced by Cohen [13, 14] (see also [5]). In particular, we consider SDEs defined by a map $\Psi: M \times N \rightarrow M$, where $M$ is the (finite dimensional) manifold where the solution lives and $N$ is the (finite dimensional) Lie group where the driving process takes values. This definition simplifies the description of the transformations of the solutions $(X, Z) \in M \times N$. In fact, if $(X, Z)$ is a solution to the SDE $\Psi(x, z)$ then, for any diffeomorphism $\Phi,(\Phi(X), Z)$ is a solution to the $\operatorname{SDE} \Phi\left(\Psi\left(\Phi^{-1}(x), z\right)\right.$ (see Theorem 2.4 and Theorem 3.1). We remark that the family of geometrical SDEs is not too restrictive: in fact it includes affine types SDEs, Marcus type SDEs, smooth SDEs driven by Lévy processes and even a class of iterated random maps (see Subsection 2.2 for further details).

The second problem is addressed by using the new notions of invariance of a semimartingale defined on a Lie group introduced in [1]. These two notions are extensions of predictable transformations which preserve the law of $n$ dimensional Brownian motion and $\alpha$-stable processes studied for example in [39, Chapter 4]. The first notion, called gauge symmetry, is a natural extension to semimartingales on Lie groups of the rotation invariance of Brownian motion, while the second one, called time symmetry, is a corresponding extension of the time rescaling invariance of Brownian motion. The concept of gauge symmetry group is based on the action $\Xi_{g}$ of a Lie group $\mathcal{G}(g$ is an element of $\mathcal{G}$ ) on the Lie group $N$ which preserves the identity $1_{N}$ of $N$. A semimartingale $Z$ admits 
$\mathcal{G}$ as gauge symmetry group if, for any locally bounded predictable process $G_{t}$ taking values in $\mathcal{G}$, the process $\tilde{Z}$, defined by the transformation $d \tilde{Z}=\Xi_{G_{t}}(d Z)$ has the same probability law as $Z$ (see Section 3). A similar definition is given for the time symmetry, where $\Xi_{g}$ is replaced by an $\mathbb{R}_{+}$action $\Gamma_{r}$ and the process $G_{t}$ is replaced by an absolutely continuous time change $\beta_{t}$ (see Section 3.2).

Given an SDE $\Psi$ and a driving process $Z$ with gauge symmetry group $\Xi_{g}$ and time symmetry $\Gamma_{r}$, we are able to define a stochastic transformation $T=(\Phi, B, \eta)$, where $\Phi, \eta$ are a diffeomorphism and a density of a time change as in the Brownian setting, while $B$ is a function taking values in $\mathcal{G}$ (in the Brownian setting $\mathcal{G}$ is the group of rotation in $\mathbb{R}^{n}$ ). In order to generalize [25], using the properties of geometrical SDEs and of gauge and time symmetries, we define an action $E_{T}$ of $T$ on the $\operatorname{SDE} \Psi$ as well as an action $P_{T}$ of $T$ on the solutions $(X, Z)$.

In this setting we propose an algorithm for exploiting general symmetries in order to reduce a geometrical SDE admitting a solvable symmetry algebra to a more simple SDE and we provide a reconstruction method to obtain solutions to the original SDE starting from the knowledge of the solutions to the reduced one.

The first novelty of our work is that, for the first time, the notion of symmetry of a SDE driven by general càdlàg, in principle non-Markovian, semimartingales is introduced and studied in full detail. The analysis is based on the introduction of a group of transformations which permits both the space transformation $\Phi$ and the gauge and time transformations $\Xi_{g}, \Gamma_{r}$. In this way our approach extends the results of [44], where only general continuous semimartingales $Z$ and space transformations $\Phi$ are considered. We also generalize the results to the case of Markovian processes on $\mathbb{R}^{m}$ with regular generators. Indeed, due to the introduction of gauge and time symmetries, we recover most of the smooth symmetries of a Markovian process which would be lost if we had just considered the space transformation $\Phi$.

The second novelty of the paper is given by our explicit approach: indeed, we provide many results which permit to check explicitly whether a semimartingale admits given gauge and time symmetries and to compute stochastic transformations which are symmetries of a given SDE. We obtain the determining equations (4.9) which are satisfied by any infinitesimal symmetry, under some mild additional hypotheses on the jumps of the driving process $Z$. The possibility of providing explicit determining equations is the main reason to restrict our attention to geometrical SDEs instead of considering more general classes of SDEs. Indeed, an interesting consequence of our study is that we provide a black-box method, applicable in several different situations, which permits to explicitly compute symmetries of a given SDE or to construct all the geometrical SDEs admitting a given symmetry. For these reasons, in order to show the generality and the user-friendliness of our theory, we conclude the paper with two applications.

The first one (see Section 5) is the study of symmetries of the general affine SDEs in two dimensions (see Section 2.2.1 for the definition of affine SDEs). This kind of model has many applications in the theory of iterated random maps (see, e.g., $[6,7,40]$ and in particular [11] for the well known ARMA model) and it is closely connected with affine processes (see [31] for the general definition of affine processes).

The second application (see Section 6) is the introduction of the general concept of weak symmetry for numerical schemes of SDEs and, in particular, for the Euler and Milstein numerical schemes for Brownian motion driven SDEs. This study is a generalization of [28], where the concept of strong symmetries of Euler and Milstein numerical schemes was introduced. Section 6, as well as [28], are parts of a more general research line, aiming at exploiting Lie symmetries of ODEs and PDEs in order to obtain better numerical integrators (see e.g. [12, 30, 46, 45] and references therein). 
The paper is organized as follows. In Section 2 we introduce the notion of geometrical SDE and we discuss their transformation properties under diffeomorphisms. In Section 3 we recall the definition of gauge and time symmetries with their main properties. In Section 4 we extend the study of the symmetries of Brownian-motion-driven SDEs to SDEs driven by general càdlàg semimartingales. Section 5 is devoted to the detailed application of our symmetries analysis to a relevant example. In Section 6 we show that the general results of the paper can be successfully applied to the most common numerical approximations for Brownian motion driven SDEs.

\section{Stochastic differential equations with jumps}

In this section we introduce some preliminary material which will be fundamental in the rest of the paper. Einstein's summation convention for repeated indices is used throughout the paper.

\subsection{Geometrical SDEs with jumps}

Definition 2.1. An adapted càdlàg stochastic process $X$ on a subset $M$ of $\mathbb{R}^{m}$ is a semimartingale if each component can be decomposed as the sum of a local martingale and a càdlàg adapted process whose sample paths are locally of bounded variation.

Semimartingales are the largest class of processes for which an Itô integral can be defined. The importance of this class relies on the fact that it is closed with respect to localization, change of time, absolutely continuous change of measure and optional stopping. Therefore, working with this class of processes allows the use of the whole standard machinery of the stochastic calculus. In particular we recall here Itô Lemma for (non continuous) semimartingales (see [52] Chapter II Section 7). If $X$ is a semimartingale on $M$ and $f$ is a twice continuously differentiable real-valued function on $M$, then $f(X)$ is a semimartingale and

$$
\begin{aligned}
f\left(X_{t}\right)= & f\left(X_{0}\right)+\int_{0}^{t} \partial_{x^{i}} f\left(X_{s^{-}}\right) d X_{s}^{i}+\frac{1}{2} \int_{0}^{t} \partial_{x^{i}, x^{j}} f\left(X_{s^{-}}\right) d\left[X^{i}, X^{j}\right]_{s}^{c} \\
& +\sum_{0 \leq s \leq t}\left\{\left(\Delta f\left(X_{s}\right)\right)-\partial_{x^{i}} f\left(X_{s^{-}}\right) \Delta X_{s}^{i}\right\},
\end{aligned}
$$

where $\Delta X_{s}=X_{s}-X_{s^{-}}$denotes the jump at time $s$ and $\left[X^{i}, X^{j}\right]^{c}$ is the continuous part of the quadratic variation defined by

$$
[X]_{t}=[X]_{t}^{c}+X_{0}^{2}+\sum_{0 \leq s \leq t}\left(\Delta X_{s}\right)^{2},
$$

where $[X]_{t}:=[X, X]_{t}$.

Hereafter, denoting by $N$ a matrix Lie group and fixing a linear representation of $N$, we denote by $z^{1}, \ldots z^{n}$ its standard set of matrix elements. For a semimartingale $Z$ on $N$, a natural definition of jump process can be given. Indeed, if $\tau$ is a stopping time, we define the jump at time $\tau$ as the random variable $\Delta Z_{\tau}$ taking values on $N$ such that

$$
\Delta Z_{\tau}=Z_{\tau} \cdot\left(Z_{\tau_{-}}\right)^{-1},
$$

where - is the multiplication in the group $N$. In order to define a special class of equations that, in some sense, depends only on the jumps $\Delta Z_{t}$ of a process $Z$ defined on $\mathrm{N}$, we consider a topological space $\mathcal{K}$ and we introduce a function $\Psi$ of the form

$$
\Psi .(\cdot, \cdot): M \times N \times \mathcal{K} \rightarrow M,
$$


such that $\Psi_{k}\left(x, 1_{N}\right)=x$ for any $k \in \mathcal{K}$ and $x \in M, \Psi_{k}$ is smooth in the $M, N$ variables and $\Psi_{k}$ and all its derivatives with respect to the $M, N$ variables are continuous in all their arguments. In order to provide a general concept of stochastic differential equation (SDE) with jumps defined on $M$ and driven by a general càdlàg semimartingale on $N$ we introduce an auxiliary function $\bar{\Psi}_{k}$

$$
\bar{\Psi}(., ., .): M \times N \times N \times \mathcal{K} \rightarrow M
$$

defined as

$$
\bar{\Psi}_{k}\left(x, z^{\prime}, z\right)=\Psi_{k}\left(x, z^{\prime} \cdot z^{-1}\right)=\Psi_{k}(x, \Delta z) .
$$

If $K$ is a predictable process such that there exist an increasing sequence of stopping times $\tau_{n} \rightarrow+\infty$ and an increasing sequence of compact sets $\mathfrak{K}_{n} \subset \mathcal{K}$ such that $K_{t}(\omega) \in \mathfrak{K}_{n}$ when $0<t \leq \tau_{n}(\omega)$, we say that $K$ is a predictable and locally bounded process. In this setting, inspired by [13], we say that the semimartingale $X$ in $M$ is a solution to the SDE defined by $\Psi_{K_{t}}$ and driven by the semimartingale $Z$ on $N$ (and we write $d X_{t}=\Psi_{K_{t}}\left(d Z_{t}\right)$ ) if and only if

$$
\begin{aligned}
X_{t}^{i}-X_{0}^{i}= & \int_{0}^{t} \partial_{z^{\prime \alpha}}\left(\bar{\Psi}_{K_{s}}^{i}\right)\left(X_{s_{-}}, Z_{s_{-}}, Z_{s_{-}}\right) d Z_{s}^{\alpha}+\frac{1}{2} \int_{0}^{t} \partial_{z^{\prime \alpha}, z^{\prime} \beta}\left(\bar{\Psi}_{K_{s}}^{i}\right)\left(X_{s_{-}}, Z_{s_{-}}, Z_{s_{-}}\right) d\left[Z^{\alpha}, Z^{\beta}\right]_{s} \\
& +\sum_{0 \leq s \leq t}\left\{\bar{\Psi}_{K_{s}}^{i}\left(X_{s_{-}}, Z_{s}, Z_{s_{-}}\right)-\bar{\Psi}_{K_{s}}^{i}\left(X_{s_{-}}, Z_{s_{-}}, Z_{s_{-}}\right)\right. \\
& \left.-\partial_{z^{\prime \alpha}}\left(\bar{\Psi}_{K_{s}}^{i}\right)\left(X_{s_{-}}, Z_{s_{-}}, Z_{s_{-}}\right) \Delta Z_{s}^{\alpha}\right\}
\end{aligned}
$$

The previous discussion can be resumed in the following

Definition 2.2. Let $K$ be a predictable locally bounded process taking values in $\mathcal{K}$. A pair of semimartingales $(X, Z)$ on $M$ and $N$ respectively is a solution to the geometrical SDE defined by $\Psi_{K_{t}}$ if $X$ and $Z$ solve the integral equation (2.4) (with $\bar{\Psi}$ given by (2.3)). When we consider $\mathcal{K}$ consisting of a single point $\left\{k_{0}\right\}$, we write $\Psi$ instead of $\Psi_{k_{0}}$.

Theorem 2.3. For any semimartingale $Z$ on $N$, there exist a stopping time $\tau$ and a semimartingale $X$ on $M$ such that $\left(X^{\tau}, Z^{\tau}\right)$ is a solution to (2.4)

Proof. The proof can be found in [13], Theorem 2.

The notion of geometrical SDE introduced in Definition 2.2 naturally suggests to consider transformations of solutions to a SDE.

Theorem 2.4. Given $M, M^{\prime} \subset \mathbb{R}^{m}$ and $N, N^{\prime}$ two matrix Lie groups, let $\Phi: M \rightarrow M^{\prime}$ and $\tilde{\Phi}: N \rightarrow N^{\prime}$ be two diffeomorphisms which respect the group action. If $(X, Z)$ is a solution to the geometrical $S D E \Psi_{K_{t}}$, then $(\Phi(X), \tilde{\Phi}(Z))$ is a solution to the geometrical $S D E \Psi_{K_{t}}^{\prime}$ defined by

$$
\bar{\Psi}_{K_{t}}^{\prime}\left(x, z^{\prime}, z\right)=\Phi\left(\bar{\Psi}_{K_{t}}\left(\Phi^{-1}(x), \tilde{\Phi}^{-1}\left(z^{\prime}\right), \tilde{\Phi}^{-1}(z)\right)\right) .
$$

Proof. The proof is based on Itô formula for semimartingales with jumps (see equation (2.1)) and the definition of geometrical SDE (2.4). The details of the proof can be found in [22], Theorem 4.3 .

\subsection{A comparison with other approaches}

The definition of geometrical SDEs driven by semimartingales with jumps includes many interesting classes of SDEs driven by càdlàg processes appearing in the literature. In particular in this section, in order to show the generality of our approach (mainly inspired by [13]), we discuss the cases of affine-type SDEs of the type studied in [52, 
Chapter V] and [9, Chapter 5] and smooth iterated random functions (see e.g. [6, 29]) that we need for our applications in Section 5 and in Section 6. Marcus-type SDEs (see $[43,49]$ ) as well as SDEs driven by Lévy processes with smooth coefficients (see e.g. $[4,42])$ can also be successfully considered in our setting: the interested reader is referred to [1] for a detailed study of these topics.

\subsubsection{Affine-type SDEs}

We briefly describe the affine type SDEs as proposed e.g. in [52, Chapter V]. In particular we show how it is possible to rewrite them according to our geometrical setting.

Let $\left(Z^{1}, \ldots, Z^{n}\right)$ be a semimartingale in $N$ and let $\sigma: M \rightarrow \operatorname{Mat}(m, n)$ be a smooth function taking values in the set of $m \times n$ matrices with real elements. We consider the SDE defined by

$$
d X_{t}^{i}=\sigma_{\alpha}^{i}\left(X_{t}\right) d Z_{t}^{\alpha},
$$

where $\sigma_{j}^{i}$ are the components of the matrix $\sigma$. If $Z_{t}^{1}=t$ and $Z^{2}, \ldots, Z^{n}$ are independent Brownian motions, we have the usual diffusion processes with drift $\left(\sigma_{1}^{1}, \ldots, \sigma_{1}^{m}\right)$ and diffusion matrix $\left.\left(\sigma_{\alpha}^{i}\right)\right|_{\substack{i=1, \ldots, m \\ \alpha=2, \ldots, n}}$.

The previous affine-type SDE can be rewritten as a geometrical SDE defined by the function $\bar{\Psi}$

$$
\bar{\Psi}\left(x, z^{\prime}, z\right)=x+\sigma(x) \cdot\left(z^{\prime}-z\right),
$$

or, in coordinates,

$$
\bar{\Psi}^{i}\left(x, z^{\prime}, z\right)=x^{i}+\sigma_{\alpha}^{i}(x)\left(z^{\prime \alpha}-z^{\alpha}\right) .
$$

In fact, by definition of geometrical SDE $\Psi$, we have

$$
\begin{aligned}
X_{t}^{i}-X_{0}^{i}= & \int_{0}^{t} \partial_{z^{\prime} \alpha}\left(\bar{\Psi}^{i}\right)\left(X_{s_{-}}, Z_{s_{-}}, Z_{s_{-}}\right) d Z_{s}^{\alpha}+\int_{0}^{t} \partial_{z^{\prime \alpha}, z^{\prime \beta}}\left(\bar{\Psi}^{i}\right)\left(X_{s_{-}}, Z_{s_{-}}, Z_{s_{-}}\right) d\left[Z^{\alpha}, Z^{\beta}\right]_{s} \\
& +\sum_{0 \leq s \leq t}\left\{\bar{\Psi}^{i}\left(X_{s_{-}}, Z_{s}, Z_{s_{-}}\right)-\bar{\Psi}^{i}\left(X_{s_{-}}, Z_{s_{-}}, Z_{s_{-}}\right)-\partial_{z^{\prime} \alpha}\left(\bar{\Psi}^{i}\right)\left(X_{s_{-}}, Z_{s_{-}}, Z_{s_{-}}\right) \Delta Z_{s}^{\alpha}\right\}, \\
= & \int_{0}^{t} \sigma_{\alpha}^{i}\left(X_{s_{-}}\right) d Z_{s}^{\alpha}+\sum_{0 \leq s \leq t}\left\{\sigma_{\alpha}^{i}\left(X_{s_{-}}\right)\left(Z_{s}^{\alpha}-Z_{s_{-}}^{\alpha}\right)-\sigma_{\alpha}^{i}\left(X_{s_{-}}\right) \Delta Z_{s}^{\alpha}\right\} \\
= & \int_{0}^{t} \sigma_{\alpha}^{i}\left(X_{s_{-}}\right) d Z_{s}^{\alpha} .
\end{aligned}
$$

\subsubsection{Iterated random smooth functions}

In the previous sections we have only considered continuous time processes $Z_{t}, t \in \mathbb{R}_{+}$. Let us now take $Z$ as a discrete time adapted process, i.e. $Z$ is a sequence of random variables $Z_{0}, Z_{1}, \ldots, Z_{l}, \ldots$ defined on $N$. We can consider $Z$ as a càdlàg continuous time process $Z_{t}$ defined as

$$
Z_{t}=Z_{l} \text { if } l \leq t<l+1, \quad t \in \mathbb{R}_{+} .
$$

Since the process $Z$ is a pure jump process with a finite number of jumps in any compact interval of $\mathbb{R}_{+}, Z$ is a semimartingale. If $(X, Z)$ is a solution to the geometrical $\operatorname{SDE} \Psi$, we have that

$$
X_{l}=\Psi\left(X_{l-1}, \Delta Z_{l}\right)
$$

and $X_{t}=X_{l}$ if $l \leq t<l+1$. The process $X$ can be viewed as a discrete time process defined by the recursive relation (2.6). These processes are special forms of iterated random functions (see e.g. $[6,29,53]$ ) and this kind of equations is very important in time series analysis (see, e.g., [11]) and in numerical simulation of SDEs (see, e.g. [41] 
for simulation of SDEs and [28] for the concept of strong symmetry of a discretization scheme). In this case we do not need that $\Psi$ is smooth in all its variables and that $\Psi\left(x, 1_{N}\right)=x$ for any $x \in M$. In the case of a discrete time semimartingale $Z_{t}$ these two conditions can be skipped and we can consider more general iterated random functions defined by relation (2.6).

An important example of iterated random functions can be obtained by considering $M=\mathbb{R}^{m}, N=G L(m) \times \mathbb{R}^{m}$ and the functions

$$
\bar{\Psi}\left(x, z^{\prime}, z\right)=\left(z_{1}^{\prime} \cdot z_{1}^{-1}\right) \cdot x+\left(z_{2}^{\prime}-z_{2}\right),
$$

where $\left(z_{1}, z_{2}\right) \in G L(m) \times \mathbb{R}^{m}$. Moreover, taking two sequences of random variables $A_{0}, \ldots, A_{l}, \ldots \in G L(n)$ and $B_{0}, \ldots, B_{l}, \ldots \in \mathbb{R}^{m}$, we define

$$
Z_{l}=\left(A_{l} \cdot A_{l-1} \cdot \ldots \cdot A_{0}, B_{l}+B_{l-1}+\ldots .+B_{0}\right) .
$$

The iterated random functions associated with the $\operatorname{SDE} \Psi$ is

$$
X_{l}=A_{l} \cdot X_{l-1}+B_{l} .
$$

This model is very well studied (see, e.g. $[6,7,40]$ ). In particular the well known ARMA model is of this form (see, e.g., [11]).

\section{Gauge and time symmetries}

In this section (see [1] for details), we generalize the well known noise change property of affine-type SDEs driven by càdlàg semimartingales. If $M=\mathbb{R}^{m}$ and $N \subseteq \mathbb{R}^{n}$, we can consider the affine SDE

$$
d X_{t}^{i}=\sigma_{\alpha}^{i}\left(X_{t_{-}}\right) d Z_{t}^{\alpha},
$$

and we can define a new semimartingale on $N$ given by

$$
d \tilde{Z}_{t}^{\alpha}=B_{\beta, t}^{\alpha} d Z_{t}^{\beta},
$$

where $B=\left(B_{\beta}^{\alpha}\right)$ is a locally bounded predictable process taking values in $G L(n)$. Therefore, the affine SDE can be rewritten in terms of the semimartingale $\tilde{Z}$ in the following way

$$
d X_{t}^{i}=\sigma_{\alpha}^{i}\left(X_{t_{-}}\right)\left(B^{-1}\right)_{\beta, t}^{\alpha} d \tilde{Z}_{t}^{\beta},
$$

where $B^{-1}$ is the inverse matrix of $B$.

The notion of geometrical SDEs allows us to introduce a useful generalization of the semimartingales change rule (3.2).

Given a Lie group $\mathcal{G}$, suppose that $M=\tilde{N}$ for some Lie group $\tilde{N}$. If we consider a smooth function

$$
\Xi .(\cdot): N \times \mathcal{G} \rightarrow \tilde{N}
$$

satisfying $\Xi_{g}\left(1_{N}\right)=1_{\tilde{N}}, \forall g \in \mathcal{G}$, we can define the map

$$
\Psi_{g}^{\Xi}(x, z)=\Xi_{g}(z) \cdot x .
$$

If $Z$ is a semimartingale on $N$ and $G$ is a predictable process taking values in $\mathcal{G}$, we define the transformed semimartingale on $\tilde{N}$ by

$$
d \tilde{Z}_{t}=\Xi_{G_{t}}\left(d Z_{t}\right)
$$

as the unique solution $(\tilde{Z}, Z)$ to the equation

$$
d \tilde{Z}_{t}=\Psi_{G_{t}}^{\Xi}\left(d Z_{t}\right),
$$


with initial condition $\tilde{Z}_{0}=1_{\tilde{N}}$. Before proving further results about transformation (3.3), we show that the semimartingales change (3.1) is a particular case of (3.3). In fact, for $\tilde{N}=N=\mathbb{R}^{n}$, any map $\Xi .: \mathbb{R}^{n} \times \mathcal{G} \rightarrow \mathbb{R}^{n}$ gives the geometrical SDE defined by the function

$$
\Psi_{g}^{\Xi}(\tilde{z}, z)=\tilde{z}+\Xi_{g}(z) .
$$

This means that equation (3.3) is explicitly given by the relation

$$
\begin{aligned}
\tilde{Z}_{t}= & \int_{0}^{t} \partial_{z^{\alpha}}\left(\Xi_{G_{s}}\right)(0) d Z_{s}^{\alpha}+\int_{0}^{t} \partial_{z^{\alpha} z^{\beta}}\left(\Xi_{G_{s}}\right)(0) d\left[Z^{\alpha}, Z^{\beta}\right]_{s}^{c}+ \\
& +\sum_{0 \leq s \leq t}\left(\Xi_{G_{s}}\left(\Delta Z_{s}\right)-\partial_{z^{\alpha}}\left(\Xi_{G_{s}}\right)(0) \Delta Z_{s}^{\alpha}\right) .
\end{aligned}
$$

If $\mathcal{G}=G L(n)$ and $\Xi_{g}(z)=\Xi_{B}(z)=B \cdot z$, since both $\partial_{z^{\alpha} z^{\beta}}\left(\Xi_{B_{t}}\right)(0)$ and $\left(\Xi_{G_{s}}\left(\Delta Z_{s}\right)-\right.$ $\left.\partial_{z^{\alpha}}\left(\Xi_{G_{s}}\right)(0) \Delta Z_{s}^{\alpha}\right)$ are equal to zero, we obtain equation (3.1).

Theorem 3.1. Let $N, \tilde{N}$ be two Lie groups and suppose that $(X, \tilde{Z})$ (where $\tilde{Z}$ is defined on $\tilde{N})$ is a solution to the geometrical SDE $\Psi_{K_{t}}$. If $d \tilde{Z}_{t}=\Xi_{G_{t}}\left(d Z_{t}\right)$, then $(X, Z)$ is a solution to the geometrical SDE defined by

$$
\hat{\Psi}_{k, g}(x, z)=\Psi_{k}\left(x, \Xi_{g}(z)\right) .
$$

Proof. For the proof see Theorem 2.8 in [1].

Corollary 3.2. Suppose that $N=\tilde{N}, \mathcal{G}$ is a Lie group and $\Xi$ is a Lie group action. If $(X, Z)$ is a solution to the geometrical $S D E \Psi_{K_{t}}$, then $(X, \tilde{Z})$ is a solution to the geometrical SDE defined by

$$
\hat{\Psi}_{k, g}(x, z)=\Psi_{k}\left(x, \Xi_{g^{-1}}(z)\right)
$$

Proof. The proof is an application of Theorem 3.1 and of the fact that $d Z_{t}=\Xi_{G_{t}^{-1}}\left(d \tilde{Z}_{t}\right)$. Indeed, defining $d \hat{Z}_{t}=\Xi_{G_{t}^{-1}}\left(d \tilde{Z}_{t}\right)$, by Theorem 3.1 we have that $d \hat{Z}_{t}=\Xi_{G_{t}^{-1}} \circ \Xi_{G_{t}}\left(d Z_{t}\right)=$ $\Xi_{1_{\mathcal{G}}}\left(d Z_{t}\right)=d Z_{t}$.

\subsection{Gauge symmetries}

Let us consider the following well known property of Brownian motion. Let $B_{t}$ : $\Omega \times[0, T] \rightarrow S O(n)$ be a predictable process taking values in the Lie group of special orthogonal matrices and consider a Brownian motion $Z$ on $\mathbb{R}^{n}$. Then the process defined by

$$
Z_{t}^{\prime \alpha}=\int_{0}^{t} B_{\beta, s}^{\alpha} d Z_{s}^{\beta}
$$

is a new $n$ dimensional Brownian motion.

In the simple case $N=\mathbb{R}^{n}$, replacing the Brownian motion by a general semimartingale, the invariance property (3.5) is no longer true. For this reason, in [1] the generalization of this property to the case of a càdlàg semimartingale $Z$ taking values in a Lie group $N$ has been proposed.

Definition 3.3. Let $Z$ be a semimartingale on a Lie group $N$ with respect to the filtration $\mathcal{F}_{t}$. Given a Lie group $\mathcal{G}$ and $g \in \mathcal{G}$, we say that $Z$ admits $\mathcal{G}$, with action $\Xi_{g}$ and with respect to the filtration $\mathcal{F}_{t}$, as gauge symmetry group if, for any $\mathcal{F}_{t}$-predictable locally bounded process $G_{t}$ taking values in $\mathcal{G}$, the semimartingale $\tilde{Z}$ solution to the equation $d \tilde{Z}_{t}=\Xi_{G_{t}}\left(d Z_{t}\right)$ has the same law as $Z$. 
In [1], in order to provide some explicit methods for checking when a semimartingale on a Lie group $N$ admits $\mathcal{G}$, with action $\Xi_{g}$, as gauge symmetry group, the concept of characteristics of a semimartingale on a Lie group has been introduced. This provides a useful condition, equivalent to Definition 3.3, that can be directly applied to Lévy processes on Lie groups in order to obtain a completely deterministic method to verify Definition 3.3 in this case. This reformulation can also be exploited to provide some examples of non-Markovian processes admitting gauge symmetry groups (see [1]).

\subsubsection{Gauge symmetries of Lévy processes}

In this section we recall the definition of a Lévy process on Lie groups with prescribed characteristics and a theorem which gives a complete characterization of gauge invariant Lévy process (see [1] for details). In order to fix notations, given $n$ right-invariant vector fields $Y_{1}, \ldots, Y_{n}$ on $N$, we introduce a set of functions $h^{1}, \ldots, h^{n}$ (called truncated functions related to $Y_{1}, \ldots, Y_{n}$ ) which are measurable, bounded, smooth in a neighborhood of the identity $1_{N}$, with compact support and such that $h^{\alpha}\left(1_{N}\right)=0$ and $Y_{\alpha}\left(h^{\beta}\right)\left(1_{N}\right)=\delta_{\alpha}^{\beta}$ (the existence of these functions is proved, for example, in [36] and they can be chosen to be equal to a set of canonical coordinates in a neighborhood of $1_{N}$ ).

Definition 3.4. Let $A_{0}$ be an $n \times n$ symmetric non-negative matrix, $b_{0}$ be an $n$-dimensional vector and $\nu_{0}$ be a positive measure such that $\int_{N}\left(h^{\alpha}(z)\right)^{2} \nu_{0}(d z)<+\infty$ and $\int_{N} f(z) \nu_{0}(d z)$ $<+\infty$ for any smooth and bounded function $f \in C^{\infty}(N)$ which is identically zero in a neighborhood of $1_{N}$. A càdlàg semimartingale $Z$ on a Lie group $N$ is called a Lévy process with characteristics $\left(b_{0}, A_{0}, \nu_{0}\right)$ if, for any $C^{2}$ function $f$ defined on $N$ and with compact support, we have that

$$
\begin{aligned}
f\left(Z_{t}\right)-\int_{0}^{t} L(f)\left(Z_{s_{-}}\right) d s: & \\
f\left(Z_{t}\right)- & \int_{0}^{t}\left(b_{0}^{\alpha} Y_{\alpha}(f)\left(Z_{s_{-}}\right)+A_{0}^{\alpha \beta} Y_{\alpha}\left(Y_{\beta}(f)\right)\left(Z_{s_{-}}\right)+\right. \\
& \left.+\int_{N}\left(f\left(z^{\prime} \cdot Z_{s_{-}}^{-1}\right)-f\left(Z_{s_{-}}\right)-h^{\alpha}\left(z^{\prime}\right) Y_{\alpha}(f)\left(Z_{s_{-}}\right)\right) \nu_{0}\left(d z^{\prime}\right)\right) d s
\end{aligned}
$$

is a local martingale.

Since the map $\Xi_{g}$ is such that $\Xi_{g}\left(1_{N}\right)=1_{N}$, using the identification of the Lie algebra generated by the right-invariant vector fields $Y_{1}, \ldots, Y_{n}$ with $\mathfrak{n}=T_{1_{N}} N$, we can define a linear maps $\Upsilon_{g}: \mathfrak{n} \rightarrow \mathfrak{n}$ in the following way

$$
\Upsilon_{g}(Y)=\left.\partial_{a}\left(\Xi_{g} \circ \Phi_{a}\right)\left(1_{N}\right)\right|_{a=0},
$$

where $Y$ is an invariant vector field with associated flow $\Phi_{a}: N \rightarrow N$. Furthermore, in order to simplify the following treatment, hereafter we require that, for any pair of flows $\Phi_{a}, \Phi_{b}^{\prime}$

$$
\left.\partial_{b}\left(\partial_{a}\left(\Xi_{g} \circ \Phi_{a} \circ \Phi_{b}^{\prime}\right)\right)\left(1_{N}\right)\right|_{a=0, b=0}=0 .
$$

This condition (which is always satisfied for the linear actions considered in this paper) can be relaxed: see [1] for the general theory.

Since $Y(f)(z)=\left.\partial_{a}\left(f \circ \Phi_{a}\right)(z)\right|_{a=0}$ and the flow of right-invariant vector fields commutes with the right multiplication, we can exploit the definition of $\Psi_{g}^{\Xi}(\tilde{z}, z)=\Xi_{g}(z) \cdot \tilde{z}$ and $\bar{\Psi}^{\Xi_{g}}\left(\tilde{z}, z^{\prime}, z\right)=\Xi_{g}\left(z^{\prime} \cdot z^{-1}\right) \cdot \tilde{z}$ to prove that, for any $Y, Y^{\prime}$ right-invariant vector fields,

$$
\begin{gathered}
Y^{z}\left(f \circ \Psi_{g}^{\Xi}\right)\left(\tilde{z}, 1_{N}\right)=Y^{z^{\prime}}\left(f \circ \bar{\Psi}_{g}^{\Xi}\right)(\tilde{z}, z, z)=\left(\Upsilon_{g}(Y)\right)(f)(\tilde{z}) \\
Y^{\prime z}\left(Y^{z}\left(f \circ \Psi_{g}^{\Xi}\right)\right)\left(\tilde{z}, 1_{N}\right)=Y^{\prime z^{\prime}}\left(Y^{z^{\prime}}\left(f \circ \bar{\Psi}_{g}^{\Xi}\right)\right)(\tilde{z}, z, z)=\left(\Upsilon_{g}\left(Y^{\prime}\right)\right)\left[\left(\Upsilon_{g}(Y)\right)(f)\right](\tilde{z})
\end{gathered}
$$


where the superscript $\cdot{ }^{z}, \cdot^{\prime}$ means that the vector fields $Y, Y^{\prime}$ apply to the $z, z^{\prime}$ variables respectively.

The following theorem gives a complete characterization of gauge invariant Lévy processes.

Theorem 3.5. If a semimartingale $Z$ is a Lévy process with characteristics $\left(b_{0}, A_{0}, \nu_{0}\right)$ such that its law is uniquely determined by its characteristics, then $Z$ admits $\mathcal{G}$ as gauge symmetry group with action $\Xi_{g}$ if and only if, for any $g \in \mathcal{G}$,

$$
\begin{aligned}
b_{0}^{\alpha} & =\Upsilon_{g, \beta}^{\alpha} b_{0}^{\beta}+\int_{N}\left(h^{\alpha}\left(z^{\prime}\right)-h^{\beta}\left(\Xi_{g^{-1}}\left(z^{\prime}\right)\right) \Upsilon_{g, \beta}^{\alpha}\right) \nu_{0}\left(d z^{\prime}\right) \\
A_{0}^{\alpha \beta} & =\Upsilon_{g, \gamma}^{\alpha} \Upsilon_{g, \delta}^{\beta} A_{0}^{\gamma \delta} \\
\nu_{0} & =\Xi_{g *}\left(\nu_{0}\right) .
\end{aligned}
$$

Remark 3.6. It is important to recall that the law of a Lévy process on the Lie group $N=\mathbb{R}^{n}$ is always uniquely determined by its characteristics (see [38], Chapter II, Theorem 4.15 and following comments).

\subsubsection{Gauge symmetries of discrete time independent increments processes}

In this section we focus on discrete time semimartingales with independent increments. We use here the convention adopted in Section 2.2.2, where we identify a discrete time process with a pure jump semimartingale having jumps at the deterministic time $t=0,1,2, \ldots, n, \ldots$.

Definition 3.7. The discrete time process $Z=\left(Z_{0}, Z_{1}, \ldots, Z_{n}, \ldots\right)$ taking values on the Lie group $N$ is a discrete time semimartingales with independent increments if $Z_{n} \cdot Z_{n-1}^{-1}$ is independent of $Z_{0}, Z_{1}, \ldots, Z_{n}$.

The law of discrete time semimartingales with independent increments is completely characterized by the measure $\nu(d z, d t)$ on $\mathbb{R}_{+} \times N$ given by

$$
\nu(d z, d t)=\sum_{n \in \mathbb{N}} \delta_{n}(d t) \mu_{n}(d z),
$$

where $\mu_{n}(d z)$ is the law of the jump $\Delta Z_{n}=Z_{n} \cdot Z_{n-1}^{-1}$.

Therefore, a semimartingale $Z^{\prime}$ has the same law as $Z$ if and only if, for any bounded continuous function on $N$,

$$
f\left(Z_{t}^{\prime}\right)-f\left(Z_{0}^{\prime}\right)=\int_{0}^{t} \int_{N} f\left(z \cdot Z_{s_{-}}^{\prime-1}\right) \nu(d z, d t) .
$$

Using the previous description of the law of $Z$ we have the following theorem.

Theorem 3.8. If a semimartingale $Z$ is a discrete time semimartingale with independent increments, then $Z$ admits $\mathcal{G}$ as gauge symmetry group with action $\Xi_{g}$ if and only if, for any $g \in \mathcal{G}$,

$$
\nu=\Xi_{g *}(\nu) .
$$

Proof. This is a particular case of Theorem 4.9 of [1].

An equivalent reformulation of the previous theorem is the following: $Z$ admits discrete time semimartingales with independent increments if and only if

$$
\Xi_{g *}\left(\mu_{n}\right)=\mu_{n}
$$


for any $n \in \mathbb{N}$. In the following we provide an interesting example, with application to iterated random maps theory, of a discrete time process admitting $O(k)$ as gauge symmetry group.

Consider $N=G L(k)$ and let $\Xi_{g}$ be the action of $O(k)$ on $N$ given by

$$
\Xi_{g}(z)=g \cdot z \cdot g^{T} .
$$

Let now $Z \in G L(k)$ be discrete-time semimartingales with independent increments characterized as follows

$$
Z_{n}=K_{n} \cdot Z_{n-1}
$$

where $K_{n} \in G L(k)$ are random variables independent of $Z_{1}, \ldots, Z_{n-1}$. Therefore, by Theorem 3.8, we have that $Z$ has $O(k)$ as gauge symmetry group with action $\Xi_{g}$ if and only if the distribution of $K_{n} \in G L(k)$ is invariant with respect to the action $\Xi_{g}$.

The invariance of the law of $K_{n} \in G L(k)$ with respect to $\Xi_{g}$ is exactly the invariance of the matrix random variable $K_{n}$ with respect to orthogonal conjugation. This kind of random variables and related processes are deeply studied in random matrix theory (see, e.g., $[3,50])$.

\subsection{Time symmetries}

In this section we briefly discuss the time symmetries of a Lévy process on a Lie group. After recalling some properties of the absolutely continuous time change, we introduce the definition of time symmetry of a semimartingale and we study time symmetries of Lévy processes, constructing some explicit examples of Lévy processes with non-trivial time symmetry.

Given a positive adapted stochastic process $\beta$ such that, for any $\omega \in \Omega$, the function $\beta(\omega): t \mapsto \beta_{t}(\omega)$ is absolutely continuous with strictly positive locally bounded derivative, we define

$$
\alpha_{t}=\inf \left\{s \mid \beta_{s}>t\right\},
$$

where, as usual, $\inf (\emptyset)=+\infty$. The process $\alpha$ is an adapted process such that

$$
\beta_{\alpha_{t}}=\alpha_{\beta_{t}}=t .
$$

If $X$ is a stochastic process adapted to the filtration $\mathcal{F}_{t}$, we denote by $H_{\beta}(X)$ the stochastic process adapted to the filtration $\mathcal{F}_{t}^{\prime}=\mathcal{F}_{\alpha_{t}}$ such that

$$
H_{\beta}(X)_{t}=X_{\alpha_{t}} .
$$

Since, by assumption, $\beta_{t}$ is absolutely continuous and strictly increasing, then also $\alpha_{t}$ is absolutely continuous and strictly increasing. Furthermore, denoting by $\beta_{t}^{\prime}$ the time derivative of $\beta_{t}$, we have

$$
\alpha_{t}^{\prime}=\frac{1}{\beta_{\alpha_{t}}^{\prime}} .
$$

If $\mu$ is a random measure on $N$ adapted to the above filtration $\mathcal{F}_{t}$, we can introduce a time changed random measure $H_{\beta}(\mu)$ adapted to the filtration $\mathcal{F}_{t}^{\prime}$ such that, for any Borel set $E \subset N$,

$$
H_{\beta}(\mu)([0, t] \times E)=\mu\left(\left[0, \alpha_{t}\right] \times E\right) .
$$

In order to introduce a good concept of symmetry with respect to time transformations, we have to recall some fundamental properties of absolutely continuous random time changes with locally bounded derivative.

Theorem 3.9. Let $\beta_{t}$ be the process described above and let $Z, Z^{\prime}$ be two real semimartingales, $K_{t}$ be a predictable process which is integrable with respect to $Z$ and $\mu$ be a random measure. Then 
1. $H_{\beta}(Z)$ is a semimartingale,

2. if $Z$ is a local $\mathcal{F}_{t}$-martingale, then $H_{\beta}(Z)$ is a local $\mathcal{F}_{t}^{\prime}$-martingale,

3. $H_{\beta}\left(\left[Z, Z^{\prime}\right]\right)=\left[H_{\beta}(Z), H_{\beta}\left(Z^{\prime}\right)\right]$

4. $H_{\beta}(K)$ is integrable with respect to $H_{\beta}(Z)$ and $\int_{0}^{\alpha_{t}} K_{s} d Z_{s}=\int_{0}^{t} H_{\beta}(K)_{s} d H_{\beta}(Z)_{s}$.

5. if $\mu^{p}$ is the compensator of $\mu$, then $H_{\beta}\left(\mu^{p}\right)$ is the compensator of $H_{\beta}(\mu)$.

Proof. Since the random time change $\beta$ is continuous, $\beta$ is an adapted change of time in the meaning of [37] (Chapter X, Section b)). Thank to this remark the proofs of assertions $1, \ldots, 5$ can be found in [37] (Chapter X, Sections b) and c)).

Taking into account Theorem 3.9, a quite natural definition of time symmetry could be the following: a semimartingale $Z$ has time symmetries if, for any $\beta$ satisfying the previous hypotheses, $Z$ and $H_{\beta}(Z)$ have the same law. Unfortunately, using for example standard deterministic time changes, it is possible to prove that the only process satisfying the previous definition is the process almost surely equal to a constant. In order to provide a different definition, admitting non-trivial examples, we introduce a smooth action $\Gamma: \mathbb{R}_{+} \times N \rightarrow N$ of the group $\mathbb{R}_{+}$on $N$. We write $\gamma_{r}: \mathfrak{n} \rightarrow \mathfrak{n}$ for the linear action of $\mathbb{R}_{+}$on $\mathfrak{n}$ such that

$$
\gamma_{r}(Y)=\left.\partial_{a}\left(\Gamma_{r} \circ \Phi_{a}\right)\left(1_{N}\right)\right|_{a=0},
$$

where $\Phi_{a}$ is the flow of a right invariant vector field $Y$ on $N$. If we assume that

$$
\left.\partial_{b}\left(\partial_{a}\left(\Gamma_{r} \circ \Phi_{a} \circ \Phi_{b}^{\prime}\right)\right)\left(1_{N}\right)\right|_{a=0, b=0}=0,
$$

we obtain

$$
\begin{gathered}
Y^{z}\left(f \circ \Psi_{r}^{\Gamma}\right)\left(\tilde{z}, 1_{N}\right)=Y^{z^{\prime}}\left(f \circ \bar{\Psi}_{r}^{\Gamma}\right)(\tilde{z}, z, z)=\left(\gamma_{r}(Y)\right)(f)(\tilde{z}) \\
Y^{\prime z}\left(Y^{z}\left(f \circ \Psi_{r}^{\Gamma}\right)\right)\left(\tilde{z}, 1_{N}\right)=Y^{\prime z^{\prime}}\left(Y^{z^{\prime}}\left(f \circ \bar{\Psi}_{r}^{\Gamma}\right)\right)(\tilde{z}, z, z)=\left(\gamma_{r}\left(Y^{\prime}\right)\right)\left[\left(\gamma_{r}(Y)\right)(f)\right](\tilde{z}) .
\end{gathered}
$$

Definition 3.10. Let $Z$ be a semimartingale on a Lie group $N$ and let $\Gamma$. $N \times \mathbb{R}_{+} \rightarrow N$ be an $\mathbb{R}_{+}$action such that $\Gamma_{r}\left(1_{N}\right)=1_{N}$ for any $r \in \mathbb{R}_{+}$. We say that $Z$ has a time symmetry with action $\Gamma_{r}$ with respect to the filtration $\mathcal{F}_{t}$ if

$$
d Z_{t}^{\prime}=H_{\beta}\left(\Gamma_{\beta_{t}^{\prime}}\left(d Z_{t}\right)\right)
$$

has the same law of $Z$ for any $\beta_{t}$ satisfying the previous hypotheses and such that $\beta_{t}^{\prime}$ is a $\mathcal{F}_{t}$-predictable locally bounded process in $\mathbb{R}_{+}$.

Remark 3.11. The request that $\beta_{t}^{\prime}$ is a locally bounded process in $\mathbb{R}_{+}$ensures that $\beta_{t}^{\prime}(\omega) \geq c(\omega)>0$ for some $c(\omega) \in \mathbb{R}_{+}$and for $t$ in compact sets.

Lemma 3.12. If $(X, Z)$ is a solution to the $S D E \Psi_{K_{t}}$ and $\beta$ is an absolutely continuous process such that $\beta_{t}^{\prime}$ is locally bounded in $\mathbb{R}_{+}$, then $\left(H_{\beta}(X), H_{\beta}(Z)\right)$ is a solution to the $S D E \Psi_{H_{\beta}(K)_{t}}$.

Proof. The thesis is a simple consequence of Definition 2.2 and Theorem 3.9, point 4.

We now restrict our attention to Lévy processes on $N$, proving a general result about Lévy processes with time symmetries.

Theorem 3.13. If $Z$ is a Lévy process with characteristics $\left(b_{0}, A_{0}, \nu_{0}\right)$, then $Z$ admits a time symmetry with action $\Gamma_{r}$ if and only if, for any fixed $r \in \mathbb{R}_{+}$,

$$
\begin{aligned}
b_{0}^{\alpha} & =\frac{1}{r}\left(\gamma_{r, \beta}^{\alpha} b_{0}^{\beta}\right)+\frac{1}{r} \int_{N}\left(h^{\alpha}\left(z^{\prime}\right)-h^{\beta}\left(\Gamma_{r^{-1}}(z)\right) \gamma_{r, \beta}^{\alpha}\right) \nu_{0}\left(d z^{\prime}\right) \\
A_{0}^{\alpha \beta} & =\frac{1}{r} \gamma_{r, \gamma}^{\alpha} \gamma_{r, \delta}^{\beta} A_{0}^{\gamma \delta} \\
\nu_{0}(d z) & =\frac{1}{r} \Gamma_{r *}\left(\nu_{0}(d z)\right) .
\end{aligned}
$$


Proof. We give the main lines of the proof. See [22], Theorem 4.36 for the details.

Put $d \tilde{Z}_{t}=H_{\beta}\left(\Gamma_{\beta^{\prime}}\left(d Z_{t}\right)\right)$. By Theorem 3.9 and by definition of stochastic characteristics (see $[1,38]$ ), the stochastic characteristics of $\tilde{Z}$ are given by

$$
\begin{aligned}
\tilde{b}_{t}^{\alpha} & =H_{\beta}\left(\int_{0}^{t}\left(\gamma_{\beta_{s}^{\prime}, \beta}^{\alpha} b_{0}^{\beta}+\int_{N}\left(h^{\alpha}\left(z^{\prime}\right)-h^{\beta}\left(\Gamma_{\beta_{s}^{\prime-1}}(z)\right) \gamma_{\beta_{s}^{\prime}, \beta}^{\alpha}\right) \nu_{0}\left(d z^{\prime}\right)\right) d s\right) \\
\tilde{A}_{t}^{\alpha \beta} & =H_{\beta}\left(\int_{0}^{t} \gamma_{\beta_{s}^{\prime}, \gamma}^{\alpha} \gamma_{\beta_{s}^{\prime}, \delta}^{\beta} A_{0}^{\gamma \delta} d s\right) \\
\tilde{\nu}(d t, d z) & =H_{\beta}\left(\Gamma_{\beta_{t}^{\prime}}\left(\nu_{0}(d z)\right) d t\right)
\end{aligned}
$$

If $\beta_{t}^{\prime}=r \in \mathbb{R}_{+}$, and so $\beta_{t}=r t$, since $\alpha_{t}=\frac{t}{r}$ we obtain

$$
\begin{aligned}
\tilde{b}_{t}^{\alpha} & =\int_{0}^{t}\left(\gamma_{r, \beta}^{\alpha} b_{0}^{\beta}+\int_{N}\left(h^{\alpha}\left(z^{\prime}\right)-h^{\beta}\left(\Gamma_{r-1}(z)\right) \gamma_{r, \beta}^{\alpha}\right) \nu_{0}\left(d z^{\prime}\right)\right) \frac{d s}{r} \\
\tilde{A}_{t}^{\alpha \beta} & =\int_{0}^{t} \gamma_{r, \gamma}^{\alpha} \gamma_{r, \delta}^{\beta} A_{0}^{\gamma \delta} \frac{d s}{r} \\
\tilde{\nu}(d t, d z) & =\frac{1}{r} \Gamma_{r, *}\left(\nu_{0}(d z)\right) d t .
\end{aligned}
$$

Since the sigma algebras generated by $Z$ and $\tilde{Z}$ coincide and so $\tilde{Z}$ has the same law of $Z$ only if $\tilde{b}_{t}=b_{0} t, \tilde{A}_{t}=A_{0} t, \tilde{\nu}(d t, d z)=\nu_{0}(d z) d t$, we get equations (3.13), (3.14) and (3.15).

Conversely suppose that equations (3.13), (3.14) and (3.15) hold and let $\beta_{t}^{\prime}$ be an elementary process. Then, by equations (3.16), (3.17) and (3.18), we have that the stochastic characteristics of $\tilde{Z}$ are exactly $b_{0} t, A_{0} t, \nu_{0}(d z) d t$. This means that $\tilde{Z}$ is a Lévy process with the same law as $Z$. Since the elementary processes are dense in the set of predictable processes, we have the thesis.

\subsection{An example of gauge and time symmetric Lévy process}

In the following using the previous theory we show that the class of $\alpha$-stable processes is an important example of time and gauge symmetric Lévy process. When $N=\mathbb{R}^{n}$ and in the homogeneous case they are well known since their generator is the fractional Laplacian, and they can be obtained by a subordination from a Brownian motion (see, e.g., $[2,4])$.

The homogeneous $\alpha$-stable processes on $N=\mathbb{R}^{n}$ are Lévy processes in $\mathbb{R}^{n}$ depending on a parameter $\alpha \in(0,2)$. The process $Z$ is a pure jump Lévy process with Lévy measure

$$
\nu_{\alpha}(d z)=\frac{1}{|z|^{n+\frac{\alpha}{2}}} d z
$$

where $|\cdot|$ is the standard norm of $\mathbb{R}^{n}, d z$ is the Lebesgue measure and $A_{0}=0, b_{0}=0$.

This means that the generator of the process $Z$ is

$$
L_{\alpha}(f)(z)=\int_{\mathbb{R}^{n}}\left(f\left(z+z^{\prime}\right)-f(z)-I_{\left|z^{\prime}\right|<1}\left(z^{\prime}\right)\left(z^{\prime \beta} \partial_{z^{\beta}}(f)(z)\right)\right) \nu_{\alpha}\left(d z^{\prime}\right) .
$$

When $B \in S O(n)$ we define

$$
\Xi_{B}(z)=B \cdot z
$$

By definition, $B$ respects the standard metric in $\mathbb{R}^{n}$ and so

$$
\Xi_{B *}\left(\nu_{\alpha}\right)=\operatorname{det}(B) \frac{1}{\left|B^{T} \cdot z\right|^{n+\frac{\alpha}{2}}} d z=\nu_{\alpha}
$$


Furthermore, since $\Upsilon_{\beta}^{\alpha}=B_{\beta}^{\alpha}$ we have

$$
\begin{aligned}
& \int_{N}\left(z^{\alpha} I_{|z|<1}(z)-\Xi_{B^{-1}}^{\beta}(z) \Upsilon_{\beta}^{\alpha} I_{\left|\Xi_{B^{-1}}(z)\right|<1}(z)\right) \nu_{\alpha}(d z)= \\
& =\int_{N}\left(z^{\alpha} I_{|z|<1}(z)-\left(B^{-1}\right)_{\gamma}^{\beta} B_{\beta}^{\alpha} I_{|z|<1}(z) z^{\gamma}\right) \nu_{\alpha}(d z)=0 .
\end{aligned}
$$

Hence, by Theorem 3.5, $Z$ admits $S O(n)$ as a gauge symmetry group with action $\Xi_{B}$.

In this case the equation $d Z_{t}^{\prime}=\Xi_{B_{t}}\left(d Z_{t}\right)$ is simply

$$
Z_{t}^{\prime \alpha}=\int_{0}^{t} B_{\beta, s}^{\alpha} d Z_{s}^{\beta} .
$$

The $\alpha$-stable processes admit also a non-trivial time symmetry. If we consider the following action

$$
\Gamma_{r}^{\alpha}(z)=r^{\frac{1}{\alpha}} z,
$$

we have that

$$
\Gamma_{r *}\left(\nu_{\alpha}\right)=\frac{1}{r} \nu_{\alpha} .
$$

Furthermore, using the fact that $\nu_{\alpha}$ is invariant with respect to rotations, we have

$$
\int_{\mathbb{R}^{n}}\left(I_{B}\left(z^{\prime}\right)-I_{\Gamma_{1 / r}(B)} z^{\prime \alpha}\right) \nu_{\alpha}\left(d z^{\prime}\right)=0 .
$$

Thus by exploiting Theorem 3.13, we obtain that the $\alpha$-stable processes are time symmetric with respect to the action $\Gamma_{r}$.

\section{Symmetry and invariance properties of a SDE with jumps}

\subsection{Stochastic transformations}

Let $\mathcal{C}\left(\mathbb{P}_{0}\right)$ (or simply $\mathcal{C}$ ) be the class of càdlàg semimartingales $Z$ on a Lie group $N$ inducing the same probability measure on $\mathcal{D}([0, T], N)$ (the metric space of càdlàg functions taking values in $N$ ). In order to generalize to the semimartingale case the notion of weak solution to an SDE driven by a Brownian motion, we introduce the following

Definition 4.1. Given a semimartingale $X$ on $M$ and a semimartingale $Z$ on $N$ such that $Z \in \mathcal{C}$, the pair $(X, Z)$ is called a process of class $\mathcal{C}$ on $M$.

A process $(X, Z)$ of class $\mathcal{C}$ which is a solution to the geometrical SDE $\Psi$ is called a solution of class $\mathcal{C}$ to $\Psi$.

We remark that if $(X, Z)$ and $\left(X^{\prime}, Z^{\prime}\right)$ are two solutions of class $\mathcal{C}$ and if $X_{0}$ and $X_{0}^{\prime}$ have the same law, then also $X$ and $X^{\prime}$ have the same law.

In this section we define a set of transformations which transform a process of class $\mathcal{C}$ into a new process of class $\mathcal{C}$. This set of transformations depends on the properties of the processes belonging to the class $\mathcal{C}$.

We start by describing the case of processes in $\mathcal{C}$ admitting a gauge-symmetry group $\mathcal{G}$ with action $\Xi_{g}$ and a time symmetry with action $\Gamma_{r}$. Afterwords, we discuss how to extend our approach to more general situations.

Definition 4.2. A stochastic transformation from a manifold $M$ into a manifold $M^{\prime}$ is a triple $(\Phi, B, \eta)$, where $\Phi$ is a diffeomorphism of $M$ into $M^{\prime}, B: M \rightarrow \mathcal{G}$ is a smooth function and $\eta: M \rightarrow \mathbb{R}_{+}$is a positive smooth function. We denote by $S\left(M, M^{\prime}\right)$ the set of stochastic transformations of $M$ into $M^{\prime}$. 
A stochastic transformation defines a map between the set of stochastic processes of class $\mathcal{C}$ on $M$ into the set of stochastic processes of class $\mathcal{C}$ on $M^{\prime}$. The action of the stochastic transformation $T \in S\left(M, M^{\prime}\right)$ on the stochastic process $(X, Z)$ is denoted by $\left(X^{\prime}, Z^{\prime}\right)=P_{T}(X, Z)$, and is defined as follows:

$$
\begin{aligned}
X^{\prime} & =\Phi\left[H_{\beta^{\eta}}(X)\right] \\
d Z_{t}^{\prime} & =H_{\beta^{\eta}}\left\{\Xi_{B\left(X_{t}\right)}\left[\Gamma_{\eta\left(X_{t}\right)}\left(d Z_{t}\right)\right]\right\}
\end{aligned}
$$

where $\beta^{\eta}$ is the random time change given by

$$
\beta_{t}^{\eta}=\int_{0}^{t} \eta\left(X_{s}\right) d s .
$$

The second step is to define an action of a stochastic transformation on the set of geometrical SDEs. This action transforms a geometrical SDE $\Psi$ on $M$ into the geometrical $\operatorname{SDE} \Psi^{\prime}=E_{T}(\Psi)$ on $M^{\prime}$ defined by

$$
\Psi^{\prime}(x, z)=\Phi\left\{\Psi\left[\Phi^{-1}(x),\left(\Gamma_{\left(\eta\left(\Phi^{-1}(x)\right)\right)^{-1}} \circ \Xi_{\left(B\left(\Phi^{-1}(x)\right)\right)^{-1}}\right)(z)\right]\right\} .
$$

Theorem 4.3. If $T \in S\left(M, M^{\prime}\right)$ is a stochastic transformation and $(X, Z)$ is a class $\mathcal{C}$ solution to the geometrical $S D E \Psi$, then $P_{T}(X, Z)$ is a class $\mathcal{C}$ solution to the geometrical $S D E E_{T}(\Psi)$.

Proof. The fact that $P_{T}(X, Z)$ is a process of class $\mathcal{C}$ follows from the symmetries of $Z$, which are the gauge symmetry group $\mathcal{G}$ with action $\Xi_{g}$ and the time symmetry with action $\Gamma_{r}$.

The fact that, if $(X, Z)$ is a solution to $\Psi$, then $P_{T}(X, Z)$ is a solution to $E_{T}(\Psi)$, follows from Theorem 2.4, Theorem 3.1 and Lemma 3.12.

Remark 4.4. If $\mathcal{C}$ contains semimartingales admitting a gauge symmetry group $\mathcal{G}$ but without time symmetry, the stochastic transformation reduces to a pair $(\Phi, B)$ and the action on processes and SDEs is the same as in the general case with $\Gamma_{r}=I d_{N}$. The same argument can be applied in the case of $\mathcal{C}$ containing semimartingales with only time symmetry.

In the case of semimartingales without neither gauge nor time symmetries, the stochastic transformations can be identified with the diffeomorphisms $\Phi: M \rightarrow M^{\prime}$ and the action on the processes is $P_{T}(X, Z)=(\Phi(X), Z)$. Since these kinds of transformations do not change the driving process $Z$ and play a special role in the theory of symmetries we call a stochastic transformation of the form $\left(\Phi, 1_{N}, 1\right)$ a strong stochastic transformation. Hereafter, in order to stress the difference between strong and more general stochastic transformations, when necessary we use the name weak stochastic transformations for the latter.

\subsection{The geometry of stochastic transformations}

In this subsection we prove that stochastic transformations have some interesting geometric properties, allowing us to extend to càdlàg-semimartingales-driven SDEs the results given in [25] for SDEs driven by Brownian motions.

In order to keep holding some crucial geometric properties, in the following we require an additional property on the maps $\Xi_{g}$ and $\Gamma_{r}$, i.e. the commutation of the two group actions $\Xi_{g}$ and $\Gamma_{r}$. In particular we suppose that

$$
\Xi_{g}\left(\Gamma_{r}(z)\right)=\Gamma_{r}\left(\Xi_{g}(z)\right),
$$

for any $z \in N, g \in \mathcal{G}$ and $r \in \mathbb{R}_{+}$. 
We can define a composition between two stochastic transformations $T \in S\left(M, M^{\prime}\right)$ and $T^{\prime} \in S\left(M^{\prime}, M^{\prime \prime}\right)$, where $T=(\Phi, B, \eta)$ and $T^{\prime}=\left(\Phi^{\prime}, B^{\prime}, \eta^{\prime}\right)$, as

$$
T^{\prime} \circ T=\left(\Phi^{\prime} \circ \Phi,\left(B^{\prime} \circ \Phi\right) \cdot B,\left(\eta^{\prime} \circ \Phi\right) \eta\right) .
$$

The above composition has a nice geometrical interpretation. If we denote by $\mathcal{H}=\mathcal{G} \times \mathbb{R}_{+}$, a stochastic transformation from $M$ into $M^{\prime}$ can be identified with an isomorphism from the trivial right principal bundle $M \times \mathcal{H}$ into the trivial right principal bundle $M^{\prime} \times \mathcal{H}$ which preserves the principal bundle structure. Exploiting this identification and the natural isomorphisms composition, we obtain formula (4.2) (see [25] for the case $\mathcal{G}=S O(m)$ ).

Given a stochastic transformation $T \in S\left(M, M^{\prime}\right)$, composition (4.2) permits to define an inverse $T^{-1} \in S\left(M^{\prime}, M\right)$ as follows

$$
T^{-1}=\left(\Phi^{-1},\left(B \circ \Phi^{-1}\right)^{-1},\left(\eta \circ \Phi^{-1}\right)^{-1}\right) .
$$

Hence the set $S(M):=S(M, M)$ is a group with respect to the composition $\circ$ and the identification of $S(M)$ with $\operatorname{Iso}(M \times \mathcal{H}, M \times \mathcal{H})$ (which is a closed subgroup of the group of diffeomorphisms of $M \times \mathcal{H}$ ) suggests to consider the corresponding Lie algebra $\mathcal{V}(M)$.

Given a one parameter group $T_{a}=\left(\Phi_{a}, B_{a}, \eta_{a}\right) \in S(M)$, there exist a vector field $Y$ on $M$, a smooth function $C: M \rightarrow \mathfrak{g}$ (where $\mathfrak{g}$ is the Lie algebra of $\mathcal{G}$ ), and a smooth function $\tau: M \rightarrow \mathbb{R}$ such that

$$
\begin{aligned}
Y(x) & :=\left.\partial_{a}\left(\Phi_{a}(x)\right)\right|_{a=0} \\
C(x) & :=\left.\partial_{a}\left(B_{a}(x)\right)\right|_{a=0} \\
\tau(x) & :=\left.\partial_{a}\left(\eta_{a}(x)\right)\right|_{a=0} .
\end{aligned}
$$

So if $Y, C, \tau$ are as above, the one parameter solution $\left(\Phi_{a}, B_{a}, \eta_{a}\right)$ to the equations

$$
\begin{aligned}
\partial_{a}\left(\Phi_{a}(x)\right) & =Y\left(\Phi_{a}(x)\right) \\
\partial_{a}\left(B_{a}(x)\right) & =R_{B_{a}(x) *}\left(C\left(\Phi_{a}(x)\right)\right) \\
\partial_{a}\left(\eta_{a}(x)\right) & =\tau\left(\Phi_{a}(x)\right) \eta_{a}(x),
\end{aligned}
$$

with initial condition $\Phi_{0}=i d_{M}, B_{0}=1_{\mathcal{G}}$ and $\eta_{0}=1$, is a one parameter group in $S(M)$. For this reason we identify the elements of $\mathcal{V}(M)$ with the triples $(Y, C, \tau)$.

Definition 4.5. A triple $V=(Y, C, \tau) \in \mathcal{V}(M)$, where $Y$ is a vector field on $M, C: M \rightarrow \mathfrak{g}$ and $\tau: M \rightarrow \mathbb{R}$ are smooth functions, is an infinitesimal stochastic transformation. If $V$ is of the form $V=(Y, 0,0)$ we call $V$ a strong infinitesimal stochastic transformation, as the corresponding one-parameter group is a group of strong stochastic transformations.

As we mentioned at the end of subsection 4.1, in order to stress the difference between strong and more general infinitesimal stochastic transformations, when necessary we use the name weak infinitesimal stochastic transformations.

Since $\mathcal{V}(M)$ is a Lie subalgebra of the set of vector fields on $M \times \mathcal{H}$, the standard Lie brackets between vector fields on $M \times \mathcal{H}$ induces some Lie brackets on $\mathcal{V}(M)$. Indeed, if $V_{1}=\left(Y_{1}, C_{1}, \tau_{1}\right), V_{2}=\left(Y_{2}, C_{2}, \tau_{2}\right) \in \mathcal{V}(M)$ are two infinitesimal stochastic transformations, we have

$$
\left[V_{1}, V_{2}\right]=\left(\left[Y_{1}, Y_{2}\right], Y_{1}\left(C_{2}\right)-Y_{2}\left(C_{2}\right)-\left\{C_{1}, C_{2}\right\}, Y_{1}\left(\tau_{2}\right)-Y_{2}\left(\tau_{1}\right)\right),
$$

where $\{\cdot, \cdot\}$ denotes the usual commutator between elements of $\mathfrak{g}$.

Furthermore the identification of $T=(\Phi, B, \eta) \in S\left(M, M^{\prime}\right)$ with $F_{T} \in \operatorname{Iso}\left(M \times \mathcal{H}, M^{\prime} \times\right.$ $\mathcal{H})$ allows us to define the push-forward $T_{*}(V)$ of $V \in \mathcal{V}(M)$ as

$$
\left(\Phi_{*}(Y),\left(A d_{B}(C)+R_{B^{-1} *}(Y(B))\right) \circ \Phi^{-1},\left(\tau+Y(\eta) \eta^{-1}\right) \circ \Phi^{-1}\right),
$$


where $A d$ denotes the adjoint operation and the symbol $Y(B)$ the push-forward of $Y$ with respect to the map $B: M \rightarrow \mathcal{G}$.

Analogously, given $V^{\prime} \in \mathcal{V}\left(M^{\prime}\right)$, we can consider the pull-back of $V^{\prime}$ defined as $T^{*}\left(V^{\prime}\right)=\left(T^{-1}\right)_{*}\left(V^{\prime}\right)$. Any Lie algebra of general infinitesimal stochastic transformations satisfying a non-degeneracy condition, can be locally transformed, by action of the push-forward of a suitable stochastic transformation $T \in S(M)$, into a Lie algebra of strong infinitesimal stochastic transformations (see Theorem 4.19 below).

\subsection{Symmetries of a SDE with jumps}

Definition 4.6. A stochastic transformation $T \in S(M)$ is a symmetry of the $S D E \Psi$ if, for any process $(X, Z)$ of class $\mathcal{C}$ solution to the $S D E \Psi$, also $P_{T}(X, Z)$ is a solution to the $S D E \Psi$.

An infinitesimal stochastic transformation $V \in \mathcal{V}(M)$ is a symmetry of the $S D E \Psi$ if the one-parameter group of stochastic transformations $T_{a}$ generated by $V$ is a group of symmetry of the SDE $\Psi$.

In the following we often use the name strong symmetries and weak symmetries for stressing the fact that the considered symmetry is a strong or a weak (infinitesimal or finite) stochastic transformation in the sense mentioned above, after Definition 4.5.

Remark 4.7. We can give also a local version of Definition 4.6: a stochastic transformation $T \in S\left(U, U^{\prime}\right)$, where $\left(U, U^{\prime}\right)$ are two open sets of $M$, is a symmetry of $\Psi$ if $P_{T}$ transforms solutions to $\left.\Psi\right|_{U}$ into solutions to $\left.\Psi\right|_{U^{\prime}}$.

In this case it is necessary to stop the solution process $X$ and the driving semimartingale $Z$ with respect to a suitably adapted stopping time.

Theorem 4.8. A sufficient condition for a stochastic transformation $T \in S(M)$ to be a symmetry of the $S D E \Psi$ is that $E_{T}(\Psi)=\Psi$.

Proof. This is an easy application of Theorem 4.3.

A natural question arising from previous discussions is whether the sufficient condition of Theorem 4.8 is also necessary. Unfortunately, even for Brownian motion driven SDEs there are counterexamples (see [25], where the determining equations for symmetries of SDE are different from the equations found here). The reason for this fact is that, for a general law of the driving semimartingale in the class $\mathcal{C}$, it is possible to find two different geometrical SDEs $\Psi$ and $\Psi^{\prime}$ with the same solution $(X, Z)$ of class $\mathcal{C}$, i.e. any solution $(X, Z)$ of $\Psi$ is also a solution of $\Psi^{\prime}$ and viceversa.

Exploiting this fact it is possible to find suitable conditions in order to prove the converse of Theorem 4.8 .

In the following we say that a semimartingale $Z$ in the class $\mathcal{C}$ and with (stochastic) characteristic triplet $(b, A, \nu)$ (see [1]) has jumps of any size if the support of $\nu$ is all of $N \times \mathbb{R}_{+}$with positive probability. If we restrict to Lévy processes this is equivalent to require that the support of $\nu_{0}$ coincides with $N$. In the case where $Z$ is a Lévy process with characteristic $\left(b_{0}, A_{0}, \nu_{0}\right)$ the previous request is equivalent to require that the support of the measure $\nu_{0}$ is all of $N$.

Lemma 4.9. Given a semimartingale $Z$ in the class $\mathcal{C}$ with jumps of any size and such that the stopping time $\tau$ of the first jump is almost surely strictly positive, if $(X, Z)$ is a solution to both the SDEs $\Psi$ and $\Psi^{\prime}$ such that $X_{0}=x_{0} \in M$ almost surely, then $\Psi\left(x_{0}, z\right)=\Psi^{\prime}\left(x_{0}, z\right)$ for any $z \in N$.

Proof. Consider the semimartingale $S_{t}^{f}=f\left(X_{t}\right)$, where $f \in C^{\infty}(M)$ is a bounded smooth function. Given a bounded smooth function $h \in C^{\infty}(\mathbb{R})$ such that $h(x)=0$ for $x$ in a 
neighborhood of 0 , we define the (special) semimartingale

$$
H_{t}^{h, f}=\sum_{0 \leq s \leq t} h\left(\Delta S_{s}^{f}\right)
$$

Since the jumps $\Delta S_{t}^{f}$ of $S^{f}$ are exactly $\Delta S_{t}^{f}=f\left(\Psi\left(X_{t_{-}}, \Delta Z_{t}\right)\right)-f\left(X_{t_{-}}\right)$or, equivalently, $\Delta S_{t}^{f}=f\left(\Psi^{\prime}\left(X_{t_{-}}, \Delta Z_{t}\right)\right)-f\left(X_{t_{-}}\right)$we have that

$$
\begin{aligned}
H_{t}^{h, f} & =\int_{N \times[0, t]} h\left(f\left(\Psi\left(X_{s_{-}}, z\right)\right)-f\left(X_{s_{-}}\right)\right) \mu^{Z}(d s, d z) \\
& =\int_{N \times[0, t]} h\left(f\left(\Psi^{\prime}\left(X_{s_{-}}, z\right)\right)-f\left(X_{s_{-}}\right)\right) \mu^{Z}(d s, d z) .
\end{aligned}
$$

Since $H^{h, f}$ is a special semimartingale there exists a unique (up to $\mathbb{P}$ null sets) predictable process $R^{h, f}$ of bounded variation such that $H_{t}^{h, f}-R_{t}^{h, f}$ is a local martingale. By the definition of characteristic measure $\nu$ it is simple to prove that

$$
\begin{aligned}
R_{t}^{h, f} & =\int_{N \times[0, t]} h\left(f\left(\Psi\left(X_{s_{-}}, z\right)\right)-f\left(X_{s_{-}}\right)\right) \nu(d s, d z) \\
& =\int_{N \times[0, t]} h\left(f\left(\Psi^{\prime}\left(X_{s_{-}}, z\right)\right)-f\left(X_{s_{-}}\right)\right) \nu(d s, d z) .
\end{aligned}
$$

This means that

$$
\int_{N \times[0, t]}\left(h\left(f\left(\Psi\left(X_{s_{-}}, z\right)\right)-f\left(X_{s_{-}}\right)\right)-h\left(f\left(\Psi^{\prime}\left(X_{s_{-}}, z\right)\right)-f\left(X_{s_{-}}\right)\right)\right) \nu(d s, d z)
$$

is a semimartingale almost surely equal to 0 . Since $X_{t_{-}}$is a continuous function for $t \leq \tau$ and the support of $\nu$ is all of $N \times \mathbb{R}_{+}$, in a set of positive measure, there exists a set of positive probability such that $h\left(f\left(\Psi\left(X_{t_{-}}, z\right)\right)-f\left(X_{t_{-}}\right)\right)-h\left(f\left(\Psi^{\prime}\left(X_{t_{-}}, z\right)\right)-f\left(X_{t_{-}}\right)\right)=0$ for any $z \in N$. Taking the limit $t \rightarrow 0$ we obtain $h\left(f\left(\Psi\left(x_{0}, z\right)\right)-f\left(x_{0}\right)\right)=h\left(f\left(\Psi^{\prime}\left(x_{0}, z\right)\right)-\right.$ $\left.f\left(x_{0}\right)\right)$. Since $h, f$ are generic functions, $\Psi\left(x_{0}, z\right)=\Psi^{\prime}\left(x_{0}, z\right)$ for any $z \in N$.

Theorem 4.10. Given a manifold $M$, under the hypothesis of Lemma 4.9, a stochastic transformation $T \in S(M)$ is a symmetry of an $S D E \Psi$ if and only if $E_{T}(\Psi)=\Psi$.

Proof. The if part is exactly Theorem 4.8 .

Conversely, suppose that $T$ is a symmetry of $\Psi$ and put $\Psi^{\prime}=E_{T}(\Psi)$. If $X^{x_{0}}$ denotes the unique solution to the $\operatorname{SDE} \Psi$ driven by the semimartingale $Z$ such that $X^{x_{0}}=x_{0}$ almost surely, put $\left(X^{\prime}, Z^{\prime}\right)=E_{T}\left(X^{x_{0}}, Z\right)$. By definition of symmetry $\left(X^{\prime}, Z^{\prime}\right)$ is a solution to $\Psi$ and, by Theorem 4.3 , it is a solution to $\Psi^{\prime}$. Since $X_{0}^{\prime}=\Phi\left(x_{0}\right)$ almost surely, using Lemma 4.9 we obtain that $\Psi\left(\Phi\left(x_{0}\right), z\right)=\Psi^{\prime}\left(\Phi\left(x_{0}\right), z\right)$. Since $\Phi$ is a diffeomorphism and $x_{0} \in M$ is a generic point this concludes the proof.

Remark 4.11. We propose here two possible generalizations of Theorem 4.10.

First we can suppose that $Z$ is a purely discontinuous semimartingale and that $b_{t}^{\alpha}=A_{t}^{\alpha, \beta}=0, \forall t \geq 0$ with truncated functions $h^{\alpha}=0$. In this case, if the support of $\nu$ is $J \times \mathbb{R}_{+}$almost surely, the stochastic transformation $T$ is a symmetry of the SDE $\Psi$ if and only if $E_{T}(\Psi)(x, z)=\Psi(x, z)$ for any $z \in J$. The proof of the necessity of the condition is equal to the one in Lemma 4.9 and Theorem 4.10, instead the proof of the sufficiency part is essentially based on the fact that $Z$ is a pure jump process. This case includes, for example, the Poisson process.

The second generalization covers the important case of continuous semimartingales. An example of the theorem which could be obtained in this case is Theorem 17 in [25] 
that, in our language, can be reformulated as follows: $T$ is a symmetry of $\Psi$ driven by a Brownian motion $Z^{2}, \ldots, Z^{m}$ and by the time $Z_{t}^{1}=t$ if and only if $\partial_{z^{\alpha}}(\Psi)(x, 0)=$ $\partial_{z^{\alpha}}\left(E_{T}(\Psi)\right)(x, 0)$ for $\alpha=2, \ldots, m$ and

$$
\partial_{z^{1}}(\Psi)(x, 0)+\frac{1}{2} \sum_{\alpha=2}^{m} \partial_{z^{\alpha} z^{\alpha}}(\Psi)(x, 0)=\partial_{z^{1}}\left(E_{T}(\Psi)\right)(x, 0)+\frac{1}{2} \sum_{\alpha=2}^{m} \partial_{z^{\alpha} z^{\alpha}}\left(E_{T}(\Psi)\right)(x, 0) .
$$

In order to provide an explicit formulation of the determining equations for the infinitesimal symmetries of a $\operatorname{SDE} \Psi$, we prove the following proposition.

Proposition 4.12. A sufficient condition for an infinitesimal stochastic transformation $V \in \mathcal{V}(M)$, generating a one-parameter group $T_{a}$ of stochastic transformations, to be an infinitesimal symmetry of an SDE $\Psi$ is that

$$
\left.\partial_{a}\left(E_{T_{a}}(\Psi)\right)\right|_{a=0}=0
$$

When the hypotheses of Theorem 4.10 hold, condition (4.7) is also necessary.

Proof. We prove that if equation (4.7) holds, then $E_{T_{a}}(\Psi)=\Psi$ for any $a \in \mathbb{R}$. Defining $\Psi(a, x, z)=E_{T_{a}}(\Psi)$, the function $\Psi(a, x, z)$ solves a partial differential equation of the form

$$
\partial_{a}(\Psi(a, x, z))=L(\Psi(a, x, z))+F(\Psi(a, x, z), x, z), \quad x \in M, z \in N
$$

where $L$ is a linear first order scalar differential operator in $\partial_{x}, \partial_{z}$ and $F$ is a smooth function. It is possible to prove, exploiting standard techniques of characteristics of first order PDE (see [25, 24]), that equation (4.8) admits a unique local solution as evolution $\mathrm{PDE}$ in the time parameter $a$ for any smooth initial value $\Psi(0, x, z)$.

Since $\Psi(0, x, z)=E_{T_{0}}(\Psi)(x, z)=\Psi(x, z)$ and $L(\Psi(x, z))+F(\Psi(x, z), x, z)=$ $\left.\partial_{a}\left(E_{T_{a}}(\Psi)\right)\right|_{a=0}=0$, we have that $E_{T_{a}}(\Psi)(x, z)=\Psi(a, x, z)=\Psi(x, z)$.

The necessity of condition (4.7) under the hypotheses of Theorem 4.10 is trivial since, by Theorem 4.10 , we must have $E_{T_{a}}(\Psi)=\Psi$.

Given a coordinate system $x^{i}$ on $M$ and $z^{\alpha}$ on $N$, we can use Proposition 4.12 to rewrite equations (4.7) in a more explicit form. If we denote by $K_{1}, \ldots, K_{r}$ the vector fields on $N$ generating the action $\Xi_{g}$ of $\mathcal{G}$ on $N$ and by $H$ the vector field generating the action $\Gamma_{r}$ of $\mathbb{R}_{+}$on $N$, with any infinitesimal stochastic transformation $V=(Y, C, \tau)$ we can associate a vector field $Y$ on $M$, a function $\tau$ and $r$ functions $C^{1}(x), \ldots, C^{r}(x)$ which correspond to the components of $C$ with respect to the basis $K_{1}, \ldots, K_{r}$. Therefore, the vector fields $Y$ and $K_{1}, \ldots, K_{r}, H$ are of the form

$$
Y=Y^{i}(x) \partial_{x^{i}} \quad K_{\ell}=K_{\ell}^{\alpha}(z) \partial_{z^{\alpha}} \quad H=H^{\alpha}(z) \partial_{z^{\alpha}}
$$

and we obtain the following theorem.

Theorem 4.13 (Determining equations). A sufficient condition such that the infinitesimal stochastic transformation $(Y, C, \tau)$ is a symmetry of the SDE $\Psi$ is that

$Y^{i}(\Psi(x, z))-Y^{j}(x) \partial_{x^{j}}\left(\Psi^{i}\right)(x, z)-\tau(x) H^{\alpha}(z) \partial_{z^{\alpha}}\left(\Psi^{i}\right)(x, z)-C^{\ell}(x) K_{\ell}^{\alpha}(z) \partial_{z^{\alpha}}\left(\Psi^{i}\right)(x, z)=0$,

where $x \in M, z \in N$ and $\Psi^{i}(x, z)=x^{i} \circ \Psi$ and $i=1, \ldots, m$. Furthermore the previous condition is also necessary if $Z$ has jumps of any size.

Proof. The proof of the necessary part of the theorem is obtained by writing the condition of Proposition 4.12 in coordinates. The sufficient part is a consequence of Theorem 4.10 . 
In the literature of symmetries on deterministic differential equations, equations (4.9) are usually called determining equations(see, e.g., [51]). It is important to note some differences with respect to the determining equations of ODEs or also of Brownianmotion-driven SDEs (see [25]). Indeed, in the deterministic case and in the Brownian motion case the determining equations are linear and local overdetermined first order differential equations both in the infinitesimal transformation coefficients and in the equation coefficients. Instead equations (4.9) are linear non-local differential equations in the coefficients $Y^{i}, \tau, C^{\ell}$ of the infinitesimal transformation $V$, and they are non-linear local differential equations in the coefficient $\Psi^{i}$ of the SDE.

\subsection{Reduction and reconstruction through infinitesimal symmetries}

In this section we propose a theorem of reduction and reconstruction for symmetric SDEs. First of all we introduce the notion of triangular SDEs.

Definition 4.14. We say that a geometrical $S D E \Psi$ is triangular with respect to the first $r$ coordinates $x^{1}, \ldots, x^{r}$ if $\Psi$ is of the form

$$
\begin{aligned}
& \Psi^{i}(x, z)=x^{i}+B^{i}\left(x^{i+1}, \ldots, x^{m}, z\right) \text { for } i=1, \ldots, r \\
& \Psi^{i}(x, z)=B^{i}\left(x^{r+1}, \ldots, x^{m}, z\right) \text { for } i=r+1, \ldots, m,
\end{aligned}
$$

for some maps $B^{i}$.

The name "triangular SDE" follows from the fact that if we write explicitly the differential relations satisfied by the processes $X_{t}^{i}$ they are triangular with respect to the process $X_{t}^{1}, \ldots, X_{t}^{r}$.

In particular this implies that the processes $X^{1}, \ldots, X^{r}$ can be reconstructed from the reduced processes $X^{r+1}, \ldots, X^{m}$ using only iterated Itô integrals. In the following we prove that if a geometrical SDE $\Psi$ admits a non-degenerate $r$ dimensional solvable Lie algebra of infinitesimal symmetries there exits a (generally local) stochastic transformation $T=(\Phi, B, \eta)$ such that $E_{T}(\Psi)$ is in triangular form. In other words we can reduce the $m$ dimensional solution $X$ to the equation $\Psi$ to a lower dimensional process $X^{\prime r+1}, \ldots, X^{\prime m}$ satisfying an $m-r$ dimensional equation and we can reconstruct the process $X$ by using only iterated Itô integrals and by inverting the stochastic transformation $T$. A consequence of this theorem is that, if $r=m$, the process $X$ can be reconstructed from a deterministic process, in other words it can be reconstructed by quadratures. These theorems can be seen as a generalization of the analogous results for symmetric deterministic ODEs (see [51]).

In order to establish these results we need some preliminary theorems and definitions.

Definition 4.15. A set of vector fields $Y_{1}, \ldots, Y_{k}$ on $M$ is regular on $M$ if, for any $x \in M$, the vectors $Y_{1}(x), \ldots, Y_{k}(x)$ are linearly independent.

Definition 4.16. Let $Y_{1}, \ldots, Y_{r}$ be a set of regular vector fields on $M$ which are generators of a solvable Lie algebra $\mathfrak{h}$. We say that $Y_{1}, \ldots, Y_{r}$ are in canonical form if there are $i_{1}, \ldots, i_{l}$ such that $i_{1}+\ldots+i_{l}=r$ and, for any $x \in M$

$$
\left(Y_{1}|\ldots| Y_{r}\right)=\left(\begin{array}{c|c|c|c}
I_{i_{1}} & G_{1}^{1}(x) & \ldots & G_{l}^{1}(x) \\
\hline 0 & I_{i_{2}} & \ldots & G_{l}^{2}(x) \\
\hline \vdots & \ddots & \ddots & \vdots \\
0 & 0 & \ldots & I_{i_{l}} \\
\hline 0 & 0 & 0 & 0
\end{array}\right)
$$

where $G_{k}^{h}: M \rightarrow \operatorname{Mat}\left(i_{h}, i_{k}\right)$ are smooth functions.

Theorem 4.17. If $\mathfrak{h}$ is an $r$-dimensional solvable Lie algebra on $M$ such that $\mathfrak{h}$ has constant dimension $r$ as a distribution of $T M$, then, for any $x_{0} \in M$, there is a set 
of generators $Y_{1}, \ldots, Y_{r}$ of $\mathfrak{h}$ and a local diffeomorphism $\Phi: U\left(x_{0}\right) \rightarrow \tilde{M}$ such that $\Phi_{*}\left(Y_{1}\right), \ldots, \Phi_{*}\left(Y_{r}\right)$ are generators in canonical form for $\Phi_{*}(\mathfrak{h})$.

Proof. The proof can be found in [24] Theorem 2.6.

Remark 4.18. In the particular case of a solvable connected Lie group $\mathcal{H}$ acting freely and regularly on $M$, Theorem 4.17 admits a (semi)-global version, i.e. we can find a coordinate system in a neighborhood of a set of orbits of the action of the group $\mathcal{H}$. Indeed, if $\mathcal{H}$ acts freely and regularly, $M$ is semi-globally diffeomorphic to $V \times \mathcal{H}$ (where $V \subset \mathbb{R}^{m-r}$ ) and the generators $Y_{1}, \ldots, Y_{r}$ of $\mathfrak{h}$ are vertical vector fields with respect to the bundle structure of $M$. Furthermore, it is possible to choose a global coordinate system $g^{1}, \ldots, g^{r}$ on $\mathcal{H}$ such that $Y_{1}, \ldots, Y_{r}$ are in canonical form (see for example [54], Chapter 2 Section 3.1 Corollary 1 ). In this way we construct a coordinate system in any neighborhood of a set of orbits of $G$.

Theorem 4.19. Let $K=\operatorname{span}\left\{V_{1}, \ldots, V_{r}\right\}$ be a Lie algebra of $\mathcal{V}(M)$ and suppose that $Y_{1}, \ldots, Y_{r}$ is a set of regular vector fields (where $V_{i}=\left(Y_{i}, C_{i}, \tau_{i}\right)$ ) and that $\mathcal{G}$ is a finite dimensional Lie algebra. Then, for any $x_{0} \in M$ there exist an open neighborhood $U$ of $x_{0}$ and a stochastic transformation $T \in S(U)$ of the form $T=\left(I d_{U}, B, \eta\right)$ such that $T_{*}\left(V_{1}\right), \ldots, T_{*}\left(V_{r}\right)$ are strong infinitesimal stochastic transformations in $\mathcal{V}(U)$. Furthermore the smooth functions $B, \eta$ are solutions to the equations

$$
\begin{aligned}
Y_{i}(B) & =-L_{B *}\left(C_{i}\right) \\
Y_{i}(\eta) & =-\tau_{i} \eta,
\end{aligned}
$$

where $L_{g}$ is the diffeomorphism given by the left multiplication for $g \in \mathcal{G}$ and $i=1, \ldots, r$.

Proof. Since $Y_{1}, \ldots, Y_{r}$ are regular vector fields for any $x_{0} \in M$ there exists a neighborhood $U$ of $x_{0}$ which is foliated by the orbits of $Y_{1}, \ldots, Y_{r}$, i.e. there exists a $M^{\prime} \subset \mathbb{R}^{m-r}$ and $H \subset \mathbb{R}^{r}$ such that $U=M^{\prime} \times H$ and such that $\left\langle Y_{1}, \ldots, Y_{r}\right\rangle \in T H$, i.e. $Y_{1}, \ldots, Y_{r}$ are vertical vector field for the trivial bundle $M^{\prime} \times H$.

Furthermore there exists a section of the trivial bundle $M^{\prime} \times H$, i.e. a smooth map $S: M^{\prime} \rightarrow U$ such that $S\left(x^{\prime}\right)=\left(x^{\prime}, S^{H}\left(x^{\prime}\right)\right)$. Let $x_{0}^{\prime} \in M^{\prime}$ be a fixed point and denote by $H_{x_{0}^{\prime}}$ the fiber of $x_{0}^{\prime}$ in the trivial bundle $M^{\prime} \times H$. Consider now the trivial bundle $H_{x_{0}^{\prime}} \times\left(\mathcal{G} \times \mathbb{R}_{+}\right)$. We can define a linear map $K: T H_{x_{0}} \rightarrow \mathfrak{g} \times \mathbb{R}$ i.e. a connection on the trivial bundle $H_{x_{0}^{\prime}} \times\left(\mathcal{G} \times \mathbb{R}_{+}\right)$using the infinitesimal stochastic transformations $V_{1}, \ldots, V_{r}$. Indeed if $R \in T H_{x_{0}^{\prime}}$, since $Y_{1}, \ldots, Y_{r}$ are regular vector fields, $R$ can be written in a unique way as $R=r^{k}(y) Y_{k}$ where $r^{k}(y)$ are smooth functions on $H_{x_{0}^{\prime}}$. Then we define $K(R)=\left(r^{k}(y) C_{k}, r^{k}(y) \tau_{k}\right)$. We prove that the connection $K$ is flat. In order to prove this it is sufficient to verify that

$$
\left[\left(Y_{i}, K\left(Y_{i}\right)\right),\left(Y_{j}, K\left(Y_{j}\right)\right)\right]=\left(\left[Y_{i}, Y_{j}\right], K\left(\left[Y_{i}, Y_{j}\right]\right)\right),
$$

where the commutation on the left hand side of the previous equation is the commutation of the vector fields $\left(Y_{k}, K\left(Y_{k}\right)\right) \in T\left(H_{x_{0}^{\prime}} \times \mathcal{G} \times \mathbb{R}_{+}\right)$. If $\left[Y_{i}, Y_{j}\right]=\lambda_{i j}^{k} Y_{k}$, by the rule of commutation of infinitesimal stochastic transformations we have

$$
\begin{aligned}
{\left[\left(Y_{i}, K\left(Y_{i}\right)\right),\left(Y_{j}, K\left(Y_{j}\right)\right)\right] } & =\left(\left[Y_{i}, Y_{j}\right], Y_{i}\left(K\left(Y_{j}\right)\right)-Y_{j}\left(K\left(Y_{i}\right)\right)-\left\{K\left(Y_{i}\right), K\left(Y_{j}\right)\right\}\right) \\
& =\left(\lambda_{i j}^{k} Y_{k},\left(Y_{i}\left(C_{j}\right)-Y_{j}\left(C_{i}\right)-\left\{C_{i}, C_{j}\right\}, Y_{i}\left(\tau_{j}\right)-Y_{j}\left(\tau_{i}\right)\right)\right) \\
& =\left(\lambda_{i j}^{k} Y_{k},\left(\lambda_{i j}^{k} C_{k}, \lambda_{i j}^{k} \tau_{k}\right)\right) \\
& =\left(\left[Y_{i}, Y_{j}\right], K\left(\left[Y_{i}, Y_{j}\right]\right)\right) .
\end{aligned}
$$

Since $K$ is a flat connection, and since without loss of generality we can suppose that $H$ is simply connected, there exists a unique function $\left(B\left(x_{0}^{\prime}, y\right), \eta\left(x_{0}^{\prime}, y\right)\right)$ defined on $H_{x_{0}^{\prime}}$ and 
solving equations (4.10) and (4.11) on $H_{x_{0}^{\prime}}$. Indeed $\left(B\left(x_{0}^{\prime}, y\right), \eta\left(x_{0}^{\prime}, y\right)\right)$ can be obtained as the parallel transport of the identity from the point $S\left(x_{0}^{\prime}\right)$ into the point $y$ through the flat connection $K$. The flatness of the connection $K$ ensures that $B\left(x_{0}^{\prime}, y\right)$ and $\eta\left(x_{0}^{\prime}, y\right)$ solve equations (4.10) and (4.11). By repeating this construction for any $x^{\prime} \in M^{\prime}$, since the section $S$ is smooth, we obtain two smooth functions $B\left(x^{\prime}, y\right)$ and $\eta\left(x^{\prime}, y\right)$ on $U=M^{\prime} \times H$ solving equations (4.10) and (4.11).

Remark 4.20. It is possible to partially extend the previous theorem to the case in which $\mathcal{G}$ is an infinite dimensional subgroup of the group of diffeomorphisms of $N$. More precisely if $\mathcal{G}_{l o c}$ is the subgroupoid of the groupoid of local diffeomorphisms of $N$ such that the Lie algebra of $\mathcal{G}_{\text {loc }}$ is generated by the Lie algebra of $\mathcal{G}$, we can prove the existence of $B \in \mathcal{G}_{\text {loc }}$ satisfying the thesis of Theorem 4.19. However sufficient conditions on $V_{1}, \ldots, V_{r}$ assuring that $B$ is not only in $\mathcal{G}_{\text {loc }}$ but also in $\mathcal{G}$ are not yet worked out.

Lemma 4.21. If $Y_{1}, \ldots, Y_{r}$ are a set of vector fields in canonical form which are also strong symmetries of the geometrical $S D E \Psi$, then $\Psi$ is in triangular form with respect to $x^{1}, \ldots, x^{r}$.

Proof. Since $Y_{1}, \ldots, Y_{r}$ are in canonical form, we have that $Y_{i}=\sum_{j<i} Y_{j}^{i}(x) \partial_{x^{j}}+\partial_{x^{i}}$. Using the determining equation for $Y_{1}$ we obtain $1-\partial_{x^{1}}\left(\Psi^{1}\right)=0$ and $\partial_{x^{1}}\left(\Psi^{i}\right)=0$ for $i>1$. This means that $\Psi^{1}=x^{1}+B^{1}\left(x^{2}, \ldots, x^{m}, z\right)$ and $\Psi^{i}$ do not depend on $x^{1}$ for $i>1$. Using an analogous reasoning and a proof by induction we can prove that $\partial_{x^{i}}\left(\Psi^{i}\right)=1$ and $\partial_{x^{i}}\left(\Psi^{k}\right)=0$ for $k>j$. This implies the thesis of the lemma.

Theorem 4.22. Let $V_{1}=\left(Y_{1}, C_{1}, \eta_{1}\right), \ldots, V_{r}=\left(Y_{r}, C_{r}, \eta_{r}\right) \in \mathcal{V}(M)$ be a solvable Lie algebra of symmetries of the $S D E \Psi$ such that $Y_{1}, \ldots, Y_{r}$ are regular vector fields and $\mathcal{G}$ is a finite dimensional Lie group. Then, for any $x_{0} \in M$, there exist a neighborhood $U$ of $x_{0}$ and a stochastic transformation $T \in S(U)$ such that $E_{T}(\Psi)$ is in triangular form.

Proof. We prove that there exists a $T=T_{1} \circ T_{2}$, where $T_{1}=\left(\Phi_{1}, i d_{\mathcal{G}}, 1\right) \in S(U)$ and $T_{2}=\left(i d_{M}, B, \eta\right) \in S(U)$, satisfying the thesis of the theorem. Owing to Theorem 4.19, the transformation $T_{2}$ can be chosen such that $T_{2}^{*}\left(V_{1}\right)=\left(Y_{1}, 0,0\right), \ldots, T_{2}^{*}\left(V_{r}\right)=\left(Y_{r}, 0,0\right)$. This means that $Y_{1}, \ldots, Y_{r}$ form a regular solvable Lie algebra of strong symmetries of $E_{T_{2}}(\Psi)$. By Theorem 4.17 there exists a (locally defined) map $\Phi_{1}$ such that $\Phi_{1}^{*}\left(Y_{1}\right), \ldots, \Phi_{1}^{*}\left(Y_{r}\right)$ are in canonical form and they are symmetries of $E_{T_{1}}\left(E_{T_{2}}(\Psi)\right)$. By Lemma 4.21, this implies that $E_{T_{1}}\left(E_{T_{2}}(\Psi)\right)$ is in triangular form. Since $E_{T}(\Psi)=E_{T_{1}}\left(E_{T_{2}}(\Psi)\right)$ the theorem is proved.

\section{A two dimensional example}

In order to give an idea of the generality and of the flexibility of our approach, in this section we propose an example of application of the previous theoretical results. For the sake of simplicity we consider only semimartingales with gauge symmetries (no time symmetries) and we use the notation introduced in Remark 4.4.

Setting $M=\mathbb{R}^{2}, N=G L(2) \times \mathbb{R}^{2}$ (with the natural multiplication), we consider the geometrical SDE

$$
\Psi\left(x, z_{(1)}, z_{(2)}\right)=z_{(1)} \cdot x+z_{(2)} .
$$

The SDE associated with $\Psi$ is an affine $\operatorname{SDE}$ and its solution $(X, Z)$ satisfies the following stochastic differential relation

$$
d X_{t}^{i}=X_{t_{-}}^{j}\left(Z_{(1)}^{-1}\right)_{j, t_{-}}^{k} d Z_{k,(1), t}^{i}+d Z_{(2), t}^{i}
$$


where $Z_{(1)}^{-1}$ is the inverse matrix of $Z_{(1)}$ and $G L(2)$ is naturally embedded in the set of the two by two matrices. If we set

$$
\bar{Z}_{j, t}^{i}=\int_{0}^{t}\left(Z_{(1)}^{-1}\right)_{j, s_{-}}^{k} d Z_{k,(1), s}^{i}
$$

equation (5.2) becomes the most general equation affine both in the noises $Z_{(1)}, Z_{(2)}$ and in the unknown process $X$. Furthermore, if the noises $Z_{(1)}, Z_{(2)}$ are discrete time semimartingales (i.e. semimartingales with fixed time jumps at times $n \in \mathbb{N}$ ) equation (5.2) becomes $X_{n}=Z_{(1), n-1}^{-1} \cdot Z_{(1), n} \cdot X_{n-1}+Z_{(2), n}-Z_{(2), n-1}$, that is an affine type iterated random map (see Subsection 2.2.2 and references therein).

The SDE $\Psi$ does not have strong symmetries, in the sense that, for general semimartingales $\left(Z_{(1)}, Z_{(2)}\right)$, equation (5.2) does not admit symmetries.

For this reason we suppose that the semimartingales $Z_{(1)}, Z_{(2)}$ have the gauge symmetry group $O(2)$ with the natural action

$$
\Xi_{B}\left(z_{(1)}, z_{(2)}\right)=\left(B \cdot z_{(1)} \cdot B^{T}, B \cdot z_{(2)}\right),
$$

where $B \in O(2)$.

In order to use the determining equation (4.9) for calculating the infinitesimal symmetries of the $\operatorname{SDE} \Psi$, we need to explicitly write the infinitesimal generator $K$ of the action $\Xi_{B}$ on $N$. In the standard coordinate system of $N$ we have that $\Xi_{B}$ is generated by

$$
\begin{aligned}
K= & \left(-z_{1,(1)}^{2}-z_{2,(1)}^{1}\right) \partial_{z_{1,(1)}^{1}}+\left(z_{1,(1)}^{1}-z_{2,(1)}^{2}\right) \partial_{z_{2,(1)}^{1}}+\left(z_{1,(1)}^{1}-z_{2,(1)}^{2}\right) \partial_{z_{1,(1)}^{2}}+ \\
& +\left(z_{2,(1)}^{1}+z_{1,(1)}^{2}\right) \partial_{z_{2,(1)}^{2}}-z_{(2)}^{2} \partial_{z_{(2)}^{1}}+z_{(2)}^{1} \partial_{z_{(2)}^{2}} .
\end{aligned}
$$

If we denote

$$
R=\left(\begin{array}{cc}
0 & -1 \\
1 & 0
\end{array}\right)
$$

we have that

$$
\begin{aligned}
& K\left(z_{(1)}\right)=R \cdot z_{(1)}+z_{(1)} \cdot R^{T} \\
& K\left(z_{(2)}\right)=R \cdot z_{(2)},
\end{aligned}
$$

where the vector field $K$ is applied componentwise to the matrix $z_{(1)}$ and the vector $z_{(2)}$. Using this property of $K$ we can easily prove that

$$
V=(Y, C)=\left(-x^{2} \partial_{x^{1}}+x^{1} \partial_{x^{2}}, 1\right),
$$

(where $C=1$ is the component of the gauge symmetry with respect to the generator $K$ ) is a symmetry of the equation $\Psi$. Indeed, recalling that $Y$ is a linear vector field whose components satisfy the relation

$$
Y=R \cdot x
$$

we have that, in this case, the determining equations (4.9) read

$$
\begin{aligned}
Y \circ \Psi-Y(\Psi)-C(x) K(\Psi)= & R \cdot\left(z_{(1)} \cdot x+z_{(2)}\right)-z_{(1)} \cdot(R \cdot x)-K(\Psi) \\
= & R \cdot\left(z_{(1)} \cdot x+z_{(2)}\right)+z_{(1)} \cdot R^{T} \cdot x-\left(R \cdot z_{(1)}+z_{(1)} \cdot R^{T}\right) \cdot x+ \\
& -R \cdot z_{(2)}=0 .
\end{aligned}
$$

Since $V$ satisfies the determining equations (4.9), $V$ is an infinitesimal symmetry of $\Psi$. The infinitesimal stochastic transformation $V$ generates a one-parameter group of symmetries of $\Psi$ given by

$$
T_{a}=\left(\Phi_{a}, B_{a}\right)=\left(\left(\begin{array}{cc}
\cos (a) & -\sin (a) \\
\sin (a) & \cos (a)
\end{array}\right) \cdot x,\left(\begin{array}{cc}
\cos (a) & -\sin (a) \\
\sin (a) & \cos (a)
\end{array}\right)\right) .
$$


In other words, if the law of $\left(Z_{(1)}, Z_{(2)}\right)$ is gauge invariant with respect to rotations, then the $\operatorname{SDE} \Psi$ is invariant with respect to rotations.

Once we have found an infinitesimal symmetry, we can exploit it to transform the SDE $\Psi$ in an equation of a simpler form as done, for example, in [24] for Brownian-motiondriven SDEs.

The first step consists in looking for a stochastic transformation $T=(\Phi, B)$ such that $T_{*}(V)$ is a strong symmetry (the existence of the transformation $T$ is guaranteed by Theorem 4.19). In this specific case the transformation $T$ has the following form

$$
T=(\Phi(x), B(x))=\left(\left(\begin{array}{l}
x^{1} \\
x^{2}
\end{array}\right),\left(\begin{array}{ll}
\frac{x^{1}}{\sqrt{\left(x^{1}\right)^{2}+\left(x^{2}\right)^{2}}} & \frac{x^{2}}{\sqrt{\left(x^{1}\right)^{2}+\left(x^{2}\right)^{2}}} \\
\frac{-x^{2}}{\sqrt{\left(x^{1}\right)^{2}+\left(x^{2}\right)^{2}}} & \frac{x^{1}}{\sqrt{\left(x^{1}\right)^{2}+\left(x^{2}\right)^{2}}}
\end{array}\right)\right)
$$

and the $\operatorname{SDE} \Psi^{\prime}=E_{T}(\Psi)$ becomes

$\Psi^{\prime}\left(x, z_{(1)}, z_{(2)}\right)=\left(\begin{array}{cc}x^{1} & -x^{2} \\ x^{2} & x^{1}\end{array}\right) \cdot\left(\begin{array}{c}z_{1,(1)}^{1} \\ z_{1,(1)}^{2}\end{array}\right)+\left(\begin{array}{cc}\frac{x^{1}}{\sqrt{\left(x^{1}\right)^{2}+\left(x^{2}\right)^{2}}} & \frac{-x^{2}}{\sqrt{\left(x^{1}\right)^{2}+\left(x^{2}\right)^{2}}} \\ \frac{x^{2}}{\sqrt{\left(x^{1}\right)^{2}+\left(x^{2}\right)^{2}}} & \frac{x^{1}}{\sqrt{\left(x^{1}\right)^{2}+\left(x^{2}\right)^{2}}}\end{array}\right) \cdot\left(\begin{array}{c}z_{(2)}^{1} \\ z_{(2)}^{2}\end{array}\right)$.

Note that $\Psi^{\prime}$ does not depend on $z_{2,(1)}^{1}, z_{2,(1)}^{2}$ and so the noise is smaller. So the transformation $T$ has an effect similar to the reduction of redundant Brownian motions in continuous SDE (see [32]). Moreover, if we rewrite the transformed SDE in (pseudo)polar coordinates

$$
\begin{aligned}
& \rho=\left(x^{1}\right)^{2}+\left(x^{2}\right)^{2} \\
& \theta=\arg \left(x^{1}, x^{2}\right),
\end{aligned}
$$

where $\arg (a, b)$ is the function giving the measure of the angle between $(0,1)$ and $(a, b)$ in $\mathbb{R}^{2}$, we find

$$
\begin{aligned}
& \Psi^{\prime \rho}(\rho, \theta, z)=\left(\sqrt{\rho} z_{1,(1)}^{1}+z_{(2)}^{1}\right)^{2}+\left(\sqrt{\rho} z_{1,(1)}^{2}+z_{(2)}^{2}\right)^{2} \\
& \Psi^{\prime \theta}(\rho, \theta, z)=\theta+\arg \left(\sqrt{\rho} z_{1,(1)}^{1}+z_{(2)}^{1}, \sqrt{\rho} z_{1,(1)}^{2}+z_{(2)}^{2}\right) .
\end{aligned}
$$

The geometrical SDE defined by $\left(\Psi^{\rho}, \Psi^{\theta}\right)$ is a triangular SDE with respect to the solutions processes $\left(R_{t}, \Theta_{t}\right)$. Indeed we have

$$
\begin{aligned}
d R_{t}= & \left(d\left[Z_{(2)}^{\prime 1}, Z_{(2)}^{\prime 1}\right]_{t}^{c}+d\left[Z_{(2)}^{\prime 2}, Z_{(2)}^{\prime 2}\right]_{t}^{c}+\left(\Delta Z_{(2), t}^{\prime 1}\right)^{2}+\left(\Delta Z_{(2), t}^{\prime 1}\right)^{2}\right)+ \\
& +\sqrt{R_{t_{-}}}\left(d Z_{(2), t}^{\prime 1}+2 d\left[Z_{(2)}^{\prime 1}, \bar{Z}_{1}^{\prime 1}\right]_{t}^{c}+2 d\left[Z_{(2)}^{\prime 2}, \bar{Z}_{1}^{\prime 2}\right]_{t}^{c}+2 \Delta \bar{Z}_{1, t}^{\prime 1} \Delta Z_{(2), t}^{\prime 1}+2 \Delta \bar{Z}_{1, t}^{\prime 2} \Delta Z_{(2), t}^{\prime 2}\right)+ \\
& +R_{t_{-}}\left(d \bar{Z}_{1, t}^{\prime 1}+d\left[\bar{Z}_{1}^{\prime 1}, \bar{Z}_{1}^{\prime 1}\right]_{t}^{c}+d\left[\bar{Z}_{1}^{\prime 2}, \bar{Z}_{1}^{\prime 2}\right]_{t}^{c}+\left(\Delta \bar{Z}_{1, t}^{1}\right)^{2}+\left(\Delta \bar{Z}_{1, t}^{2}\right)^{2}\right) \\
d \Theta_{t}= & \left(-d \bar{Z}_{1, t}^{\prime 2}+2 d\left[\bar{Z}_{1}^{\prime 1}, \bar{Z}_{1}^{\prime 2}\right]_{t}^{c}\right)+ \\
& +\frac{1}{\sqrt{R_{t_{-}}}}\left(-d Z_{(2), t}^{\prime 2}+2 d\left[\bar{Z}_{1}^{\prime 1}, Z_{(2)}^{\prime 2}\right]_{t}^{c}+2 d\left[Z_{(2)}^{\prime 1}, \bar{Z}_{1}^{\prime 2}\right]_{t}^{c}\right)+\frac{2}{R_{t_{-}}} d\left[Z_{(2)}^{\prime 1}, Z_{(2)}^{\prime 2}\right]_{t}^{c}+ \\
& +\left(\arg \left(\sqrt{R_{t_{-}}}\left(1+\Delta \bar{Z}_{1, t}^{\prime 1}\right)+\Delta Z_{(2), t}^{\prime 1}, \sqrt{R_{t_{-}}}\left(\Delta \bar{Z}_{1, t}^{\prime 2}\right)+\Delta Z_{(2), t}^{\prime 2}\right)+\Delta \bar{Z}_{1, t}^{\prime 2}+\frac{\Delta Z_{(2), t}^{\prime 2}}{\sqrt{R_{t_{-}}}}\right),
\end{aligned}
$$

where

$$
\begin{aligned}
d Z_{(2), t}^{\prime i} & =B_{j}^{i}\left(X_{t_{-}}\right) d Z_{(2), t}^{j} \\
d Z_{j,(1), t}^{\prime i} & =Z_{k(1), t_{-}}^{\prime i} B_{l}^{k}\left(X_{t_{-}}\right) B_{r}^{j}\left(X_{t_{-}}\right)\left(Z_{(1)}^{-1}\right)_{m, t_{-}}^{l} d Z_{r,(1), t}^{m} \\
d \bar{Z}_{j, t}^{\prime i} & =\left(Z_{(1)}^{\prime-1}\right)_{k, t_{-}}^{i} d Z_{j(1), t}^{\prime k}=B_{k}^{i}\left(X_{t_{-}}\right) B_{r}^{j}\left(X_{t_{-}}\right) d \bar{Z}_{r, t}^{k} .
\end{aligned}
$$


Here $B(x)$ is given in (5.5), $X_{t}^{1}=\sqrt{R_{t}} \cos \left(\Theta_{t}\right), X_{t}^{2}=\sqrt{R_{t}} \sin \left(\Theta_{t}\right)$ and $\bar{Z}_{j}^{i}$ are given in equation (5.3). SDEs (5.7) and (5.8) are in triangular form: indeed, the equation for $R_{t}$ depends only on $R_{t}$, while the equation for $\Theta_{t}$ is independent of $\Theta_{t}$ itself. This means that the process $\Theta_{t}$ can be reconstructed from the process $R_{t}$ and the semimartingales $\left(Z_{(1)}^{\prime}, Z_{(2)}^{\prime}\right)$ using only integrations. Furthermore, using the inverse of the stochastic transformation (5.5), we can recover both the solution process $X_{t}^{1}, X_{t}^{2}$ and the initial noise $\left(Z_{(1)}, Z_{(2)}\right)$ using only inversion of functions and Itô integrations. This situation is very similar to what happens in the deterministic setting (see [51]) and in the Brownian motion case (see [24]), where the presence of a one-parameter symmetry group allows us to split the differential system into a system of lower dimension and an integration (the so called reduction and reconstruction by quadratures). Also the equation for $R_{t}$ seems to have a familiar form. Indeed, if $Z_{(1)}=I_{2}$ (the two dimensional identity matrix) and $Z_{(2)}$ is a two dimensional Brownian motion, equation (5.7) becomes the equation of the two dimensional Bessel process. This fact should not surprise since the proposed reduction procedure is the usual reduction procedure of a two dimensional Brownian motion with respect to the rotation group. For generic $\left(Z_{(1)}, Z_{(2)}\right)$ the equation for $R_{t}$ has the form

$$
d R_{t}=d \mathfrak{Z}_{t}^{1}+\sqrt{R_{t_{-}}} d \mathfrak{Z}_{t}^{2}+R_{t_{-}} d \mathfrak{Z}_{t}^{2}
$$

for suitable semimartingales $\mathfrak{Z}^{1}, \mathfrak{Z}^{2}$ and $\mathfrak{Z}^{3}$. Equation (5.7) can be considered a kind of generalization of affine processes (see [31]): indeed, if $Z_{(1)}$ is deterministic and $Z_{(2)}$ is a two dimensional Brownian motion, equation (5.7) reduces to a CIR model equation with time dependent coefficients.

\section{Weak symmetries of numerical approximations of SDEs driven by Brownian motion}

In this last section we deal with the symmetries of the numerical schemes of Brownianmotion driven SDEs as studied in [28], where an invariant numerical integrator for symmetric Brownian motion driven SDEs was introduced by means of the identification of a suitable coordinate system for the discretization. Indeed, in [28] the concept of strong symmetry of an SDE and of the related numerical scheme was exploited in order to provide necessary and sufficient conditions guaranteeing invariance properties of the adapted-to-symmetries numerical approximations. Moreover, some theoretical estimates showing the stability and efficiency of the symmetry methods for the class of general linear SDEs were proved.

In this section we extend the results of [28] defining the concept of weak symmetry of a numerical discretization of a Brownian-motion-driven SDE. Using the theory described in this paper, in the notations of Remark 4.4, a weak symmetry of a numerical discretization of a Brownian-motion-driven SDE is a pair $(\Phi, B)$ where $B \in O(k)$ is a gauge symmetry.

On the other hand, there is a natural notion of (weak) symmetry for Brownianmotion-driven SDEs (see [25]) and in the following we discuss the relations between the symmetries of the SDE and the (weak) symmetries of its discretization.

After recalling the notion of (weak) symmetry for Brownian-motion-driven SDEs, we exploit the general theory developed in this paper to describe a numerical scheme for a Brownian-motion-driven SDE as a geometrical SDE driven by a semimartingale $Z$ related to the Brownian motion $W$. Once the gauge symmetries of the semimartingale $Z$ are analyzed, we are able to find the symmetries of the numerical schemes as the symmetries of the geometrical SDE.

In particular we focus on the two more common numerical schemes for equation (6.1): the Euler and the Milstein discretizations. 


\subsection{Symmetries of Brownian-motion-driven SDEs}

In this section we recall the notion of weak symmetry for a Brownian-motion-driven SDE as developed in [25]. This notion can be seen as a special case of the theory described in this paper. The fundamental difference is that, in the Brownian motion case, the determining equations (4.9) give only a sufficient condition for finding symmetries. This is due to the fact that, since Brownian motion is a continuous semimartingale, there is a huge set of geometrical SDEs $\Psi$ driven by Brownian motion having the same set of solutions.

For this reason hereafter an SDE driven by the Brownian motion is no more identified with a geometrical SDE $\Psi$ but with the more standard pair of coefficients $(\mu, \sigma)$. More precisely the $\operatorname{SDE}(\mu, \sigma)$ is an equation of the form

$$
d X_{t}^{i}=\mu^{i}\left(X_{t}\right) d t+\sigma_{\alpha}^{i}\left(X_{t}\right) d W_{t}^{\alpha}
$$

for $\alpha=1, \ldots, k$. A solution $X$ to the $\operatorname{SDE}(6.1)$ is a diffusion process admitting as infinitesimal generator:

$$
L=\frac{1}{2} \sigma_{\alpha}^{i} \sigma_{\alpha}^{j} \partial_{x^{i} x^{j}}+\mu^{i} \partial_{x^{i}}
$$

Since Brownian motion has both gauge symmetries (given by the rotations group $O(k)$ with its natural action on $\mathbb{R}^{k}$ ) and time symmetries (with action $\Gamma_{r}(z)=r^{1 / 2} z$ ), a weak (infinitesimal) symmetry is given by a triplet $(Y, C, \tau)$ with $C: M \rightarrow \mathfrak{s o}(k)$ and $\eta: M \rightarrow \mathbb{R}$. We first recall the following theorem.

Theorem 6.1. Let $V=(Y, C, \tau)$ be an infinitesimal stochastic transformation. Then $V$ is a weak symmetry of the $S D E(\mu, \sigma)$ if and only if $Y$ generates a one parameter group on $M$ and the following determining equations hold

$$
\begin{gathered}
Y^{i} \partial_{x^{i}}\left(\mu^{j}\right)-L\left(Y^{j}\right)+\tau \mu^{j}=0 \\
Y^{i} \partial_{x^{i}}\left(\sigma_{\alpha}^{j}\right)-\sigma_{\alpha}^{i} \partial_{x^{i}}\left(Y^{j}\right)+\frac{1}{2} \tau \sigma_{\alpha}^{i}+\sigma_{\beta}^{j} C_{\alpha}^{\beta}=0 .
\end{gathered}
$$

Proof. The proof can be found in [25], Theorem 4.4.

In the following we use the name quasi strong (infinitesimal) transformations or symmetries for stochastic transformations $V$ of the form $V=(Y, C, 0)$.

\subsection{Symmetries of the Euler scheme}

The Euler scheme for equation (6.1), given a partition $\left\{t_{\ell}\right\}_{\ell}$ of $[0, T]$, reads

$$
X_{t_{\ell}}^{i, N}=X_{t_{\ell-1}}^{i, N}+\mu\left(X_{t_{\ell-1}}^{N}\right) \Delta t_{\ell}+\sum_{\alpha=1}^{k} \sigma_{\alpha}^{i}\left(X_{t_{\ell-1}}^{N}\right) \Delta W_{\ell}^{\alpha}
$$

where $i=1, \ldots, n, \Delta t_{\ell}=t_{\ell}-t_{\ell-1}$ and $\Delta W_{\ell}^{\alpha}=W_{t_{\ell}}^{\alpha}-W_{t_{\ell-1}}^{\alpha}$. We define a semimartingale $Z$ taking values in $N=\mathbb{R}^{k+1}$ by putting

$$
\begin{aligned}
Z_{t}^{0} & =\sum_{\ell=0}^{\infty} t_{\ell} I_{\left[t_{\ell}, t_{\ell+1}\right)}(t) \\
Z_{t}^{\alpha} & =\sum_{\ell=0}^{\infty} W_{t_{\ell}}^{\alpha} I_{\left[t_{\ell}, t_{\ell+1}\right)}(t) .
\end{aligned}
$$

Since $Z_{t}$ is a process with predictable jumps at $t_{\ell}, Z_{t}$ is a semimartingale. Let us introduce the maps

$$
F^{i}(x, z)=x^{i}+\mu(x) z^{0}+\sigma_{\alpha}^{i}(x) z^{\alpha} .
$$


It is clear that $X_{t}^{N}$, solution to (6.4), can be seen as the solution to the geometrical SDE defined by $F$ and driven by the above defined semimartingale $Z$ (according to (2.4) with $\left.\Psi^{i}=F^{i}\right)$.

Let $\Xi_{B}$ be an action of $O(k)$ on $\mathbb{R}^{k+1}$ such that $\Xi_{B}(z)=\left(z^{0}, B_{\beta}^{\alpha} z^{\beta}\right)$, where, $B_{\beta}^{\alpha}$ is the $\alpha, \beta$ element of a square matrix of dimension $k$. The following theorem holds.

Theorem 6.2. The group $O(k)$ with action $\Xi_{B}$ is a gauge symmetry group for $Z$ with respect to its natural filtration.

Proof. We directly use the definition of gauge symmetry group. Let $\mathcal{F}_{t}^{W}$ be the natural filtration of the $k$ dimensional Brownian motion $W$. Let $B_{t}$ be a predictable locally bounded process taking values in $O(k)$ with respect to the natural filtration of $Z \mathcal{F}_{t}^{Z}$. This means that $B_{t_{\ell}}$ is $\mathcal{F}_{t_{\ell-1}}^{W}$ measurable. Moreover, the process $\tilde{B}_{t}$, defined by

$$
\tilde{B}_{t}=\sum_{\ell=0}^{\infty} B_{t_{\ell}} I_{\left(t_{\ell-1}, t_{\ell}\right]}(t)
$$

is an $\mathcal{F}_{t}^{W}$-predictable locally bounded process taking values in $O(k)$. Since the rotations are a gauge symmetry group for the $k$ dimensional Brownian motion with respect to its natural filtration, we have that

$$
\tilde{W}_{t}^{\alpha}=\int_{0}^{t} \tilde{B}_{\beta, s}^{\alpha} d W_{s}^{\beta}=\sum_{\ell=1}^{+\infty} B_{\beta, t_{\ell}}^{\alpha}\left(W_{t_{\ell} \wedge t}^{\beta}-W_{t_{\ell-1} \wedge t}^{\beta}\right)
$$

is a $k$ dimensional Brownian motion. In particular the following discretization

$$
\tilde{Z}_{t}^{\alpha}=\sum_{\ell=0}^{\infty} \tilde{W}_{t_{\ell}}^{\alpha} I_{\left[t_{\ell}, t_{\ell+1}\right)}(t)
$$

has the same law as $Z$ (as can be immediately seen from (6.5) and (6.6)). Since

$$
\begin{aligned}
\tilde{Z}_{t_{i}}^{\alpha} & =\int_{0}^{t_{i}} B_{\beta, s}^{\alpha} d Z_{s}^{\beta}=\sum_{\ell=1}^{i} B_{\beta, t_{\ell}}^{\alpha}\left(Z_{t_{\ell-1}}^{\beta}-Z_{t_{\ell}}^{\beta}\right) \\
& =\sum_{\ell=1}^{i} B_{\beta, t_{\ell}}^{\alpha}\left(W_{t_{\ell}}^{\beta}-W_{t_{\ell-1}}^{\beta}\right)=\tilde{W}_{t_{i}}^{\alpha}
\end{aligned}
$$

and $B_{t}$ is a generic predictable locally bounded process with respect to the filtration $\mathcal{F}_{t}^{Z}$, generated by $Z$, the thesis follows.

Using the previous discussion and Theorem 6.2 we can introduce the concept of weak symmetry of the Euler discretization scheme $F(x, z)$. Indeed the weak stochastic transformation $T=(\Phi(x), B(x))$, which acts on the solution to the Euler discretization scheme in the following way $\left(X^{\prime N}, Z^{\prime}\right)=P_{T}(X, Z)$ and precisely as

$$
\begin{aligned}
X_{t_{\ell}}^{\prime N} & =\Phi\left(X_{t_{\ell}}^{N}\right) \\
\Delta Z_{t_{\ell}}^{\prime \alpha} & =B_{\beta}^{\alpha}\left(X_{t_{\ell-1}}^{N}\right) \Delta Z_{t_{\ell}}^{\beta}=B_{\beta}^{\alpha}\left(X_{t_{\ell-1}}^{N}\right) \Delta W_{\ell}^{\beta} \\
\Delta Z_{t_{\ell}}^{0} & =\Delta t_{\ell},
\end{aligned}
$$

is a symmetry of the Euler discretization scheme if $\left(X^{\prime}, Z^{\prime}\right)$ is also a solution to the discretization scheme defined by $F$. By Theorem 4.8, a sufficient condition to be a symmetry of $F$ is

$$
\Phi\left(F\left(\Phi^{-1}(x), \Delta t,\left(B \circ \Phi^{-1}(x)\right)^{-1} \cdot \Delta W\right)=F(x, \Delta t, \Delta W),\right.
$$


where $z=(\Delta t, \Delta W)$. For a given weak infinitesimal stochastic transformation $(Y, C)$ the determining equations reads

$$
\begin{aligned}
& Y^{j}(x) \partial_{x^{j}}\left(F^{i}\right)(x, \Delta t, \Delta W)-F^{j}(x, \Delta t, \Delta W) \partial_{x^{j}}\left(Y^{i}\right)(x) \\
& \quad=-C_{\beta}^{\alpha}(x) \Delta W^{\beta} \partial_{\Delta W^{\alpha}}\left(F^{i}\right)(x, \Delta t, \Delta W) .
\end{aligned}
$$

The following theorem proposes a generalization of Theorem 3.1 in [28] to the case of weak stochastic transformations.

Theorem 6.3. Let $V=(Y, C, 0)$ be a quasi strong symmetry of the SDE (6.1). When $Y_{j}^{i}=Y_{j}\left(x^{i}\right)$ are polynomials of first degree in $x^{1}, \ldots, x^{m}$, then $\tilde{V}=(Y, C) \in \mathcal{V}(M)$ is a weak symmetry of the Euler discretization scheme $F$. If, for a given $x_{0} \in M$, $\operatorname{span}\left\{\sigma_{1}\left(x_{0}\right), \ldots, \sigma_{m}\left(x_{0}\right)\right\}=\mathbb{R}^{n}$, also the converse holds.

Proof. The determining equations for the special case of the Euler discretization method are

$$
Y^{i}\left(x+\mu \Delta t+\sigma_{\alpha} \Delta W^{\alpha}\right)-Y^{i}-Y^{j} \partial_{x^{j}}\left(\mu^{i}\right) \Delta t-Y^{j} \partial_{x^{j}}\left(\sigma_{\alpha}^{i}\right) \Delta W^{\alpha}-\sigma_{\beta}^{i} C_{\alpha}^{\beta} \Delta W^{\alpha}=0 .
$$

Using equations (6.3) in equation (6.9) we obtain

$$
Y^{i}\left(x+\mu \Delta t+\sigma_{\alpha} \Delta W^{\alpha}\right)=Y^{i}+\mu^{j} \partial_{x^{j}}\left(Y^{i}\right) \Delta t+A^{l k} \partial_{x^{k} x^{l}}\left(Y^{i}\right) \Delta t+\sigma_{\alpha}^{j} \partial_{x^{j}}\left(Y^{i}\right) \Delta W^{\alpha} .
$$

If $Y^{i}$ is linear in $x^{j}$, equation (6.10) is satisfied and $(Y, C)$ is a weak symmetry of the Euler discretization scheme. Conversely, if $(Y, C)$ is a symmetry of the Euler discretization scheme and the condition assumed for $\sigma_{\alpha}$ holds, equation (6.10) implies that $Y^{i}$ is linear in $x^{j}$.

\subsection{Symmetries of the Milstein scheme}

The Milstein scheme has the form

$$
\begin{aligned}
\bar{X}_{t_{\ell}}^{i, N}= & \bar{X}_{t_{\ell-1}}^{i, N}+\mu^{i}\left(\bar{X}_{t_{\ell-1}}\right) \Delta t_{\ell}+\sum_{\alpha=1}^{k} \sigma_{\alpha}^{i}\left(\bar{X}_{t_{\ell-1}}\right) \Delta W_{\ell}^{\alpha}+ \\
& +\frac{1}{2} \sum_{j=1}^{m} \sum_{\alpha, \beta=1}^{k} \sigma_{\alpha}^{j}\left(\bar{X}_{t_{\ell-1}}\right) \partial_{j}\left(\sigma_{\beta}^{i}\right)\left(\bar{X}_{t_{\ell-1}}\right) \Delta W_{\ell}^{\alpha, \beta}
\end{aligned}
$$

where $\Delta \mathbb{W}_{\ell}^{\alpha, \beta}=\int_{t_{\ell-1}}^{t_{\ell}}\left(W_{s}^{\beta}-W_{t_{\ell-1}}^{\beta}\right) d W_{s}^{\alpha}$. We recall that when $n=1$ we have that

$$
\Delta W_{\ell}^{1,1}=\frac{1}{2}\left(\left(\Delta W_{\ell}\right)^{2}-\Delta t_{\ell}\right)
$$

We cannot consider the scheme as a geometrical SDE driven by a semimartingale in $\mathbb{R}^{k+1}$ anymore because in this case the driving noise is composed by both the discretization of the Brownian motion $W$ and by the iterated integral $W_{t}^{\alpha, \beta}=\int_{0}^{t} W_{s}^{\alpha} d W_{s}^{\beta}$. Therefore in this case

$$
N=\mathbb{R} \oplus \mathbb{R}^{k} \oplus\left(\mathbb{R}^{k} \otimes \mathbb{R}^{k}\right),
$$

and the semimartingale $\left(t, W_{t}, \mathbb{W}_{t}\right)$ lives exactly in $N$. The vector space $N$ has a natural Lie group structure with composition given by

$$
\left(\alpha_{1}, a_{1}, b_{1}\right) \circ\left(\alpha_{2}, a_{2}, b_{2}\right)=\left(\alpha_{1}+\alpha_{2}, a_{1}+a_{2}, a_{2} \otimes a_{1}+b_{1}+b_{2}\right),
$$

where $\alpha_{1}, \alpha_{2} \in \mathbb{R}, a_{1}, a_{2} \in \mathbb{R}^{k}$ and $b_{1}, b_{2} \in \mathbb{R}^{k} \otimes \mathbb{R}^{k}$. In this case $1_{N}=(0,0,0 \otimes 0)$, while the inverse operation is given by

$$
\left(\alpha_{1}, a_{1}, b_{1}\right)^{-1}=\left(-\alpha_{1},-a_{1},-b_{1}+a_{1} \otimes a_{1}\right) .
$$


Let $Z=\left(Z^{0}, Z_{1}^{\alpha}, Z_{2}^{\alpha, \beta}\right)$ be the semimartingale given by the discretization of $\left(t, W_{t}, \mathbb{W}_{t}\right)$, that is

$$
\begin{aligned}
Z_{t}^{0} & =t_{\ell} \text { if } t_{\ell} \leq t<t_{\ell+1} \\
Z_{1, t}^{\alpha} & =W_{t_{\ell}}^{\alpha} \text { if } t_{\ell} \leq t<t_{\ell+1} \\
Z_{2, t}^{\alpha, \beta} & =\int_{0}^{t_{\ell}} W_{s}^{\alpha} d W_{s}^{\beta} \text { if } t_{\ell} \leq t<t_{\ell+1} .
\end{aligned}
$$

It is simple to see that

$$
\begin{aligned}
Z_{t_{\ell}} \circ Z_{t_{\ell-1}}^{-1}= & \left(t_{\ell}-t_{\ell-1}, W_{t_{\ell}}^{\alpha}-W_{t_{\ell-1}}^{\alpha}, \int_{0}^{t_{\ell}} W_{s}^{\alpha} d W_{s}^{\beta}+\right. \\
& \left.-\int_{0}^{t_{\ell-1}} W_{s}^{\alpha} d W_{s}^{\beta}-W_{t_{\ell-1}}^{\alpha} W_{t_{\ell}}^{\beta}+W_{t_{\ell-1}}^{\alpha} W_{t_{\ell-1}}^{\beta}\right) \\
= & \left(\Delta t_{\ell}, \Delta W_{\ell}^{\alpha}, \Delta W_{\ell}^{\alpha \beta}\right) .
\end{aligned}
$$

It is possible to define, in a natural way, an action $\Xi_{B}: N \rightarrow N$ of the orthogonal matrices $B \in O(k)$ on $N$. Indeed if $B \in N$ we define $\Xi_{B}$ as the unique linear map from $N$ into itself such that

$$
\Xi_{B}((\alpha, a, b \otimes c))=(\alpha, B \cdot a,(B \cdot b) \otimes(B \cdot c)),
$$

for any $a, b, c \in \mathbb{R}^{k}$.

Theorem 6.4. The Lie group $O(k)$ with action $\Xi_{B}$ is a gauge symmetry group for the discretization $Z=\left(Z^{0}, Z_{1}^{\alpha}, Z_{2}^{\alpha, \beta}\right)$ of $\left(t, W_{t}, W_{t}\right)$, with respect to its natural filtration.

Proof. Let us define

$$
W_{t}^{\prime}=\sum_{t_{k} \leq t} B_{t_{k}} \cdot \Delta W_{k}+B_{t_{\ell}}\left(W_{t}-W_{t_{\ell}}\right),
$$

where $t_{k}$ is the last discrete time lower than $t$. The thesis of the theorem is equivalent to prove that

$$
\mathrm{W}_{t_{\ell}}^{\prime \alpha \beta}=\int_{0}^{t_{\ell}} W_{s}^{\prime \alpha} d W_{s}^{\prime \beta}=Z_{2, t_{\ell}}^{\prime \alpha \beta}
$$

where $d Z_{t}^{\prime}=\Xi_{B}\left(d Z_{t}\right)$, that is, in particular, the third component of $Z_{t_{\ell}}^{\prime}$ is given by

$$
\begin{aligned}
Z_{2, t_{\ell}}^{\prime \alpha \beta} & =\sum_{k \leq \ell} B_{\gamma, t_{k}}^{\alpha} B_{\delta, t_{k}}^{\beta} \Delta Z_{2, t_{k}}^{\gamma \delta}+\sum_{h \leq k<\ell} B_{\gamma, t_{h}}^{\alpha} B_{\delta, t_{k}}^{\beta} \Delta Z_{1, t_{h}}^{\gamma} \Delta Z_{1, t_{k}}^{\delta} \\
& =\sum_{k \leq \ell} B_{\gamma, t_{k}}^{\alpha} B_{\delta, t_{k}}^{\beta} \Delta W_{k}^{\gamma \delta}+\sum_{h \leq k<\ell} B_{\gamma, t_{h}}^{\alpha} B_{\delta, t_{k}}^{\beta} \Delta W_{h}^{\gamma} \Delta W_{k}^{\delta} .
\end{aligned}
$$

We have that

$$
\begin{aligned}
\mathbb{W}_{t_{\ell}}^{\prime \alpha \beta} & =\int_{0}^{t_{\ell}} W_{s}^{\prime \alpha} d W_{s}^{\prime \beta} \\
& =\int_{0}^{t_{\ell}}\left(\sum_{t_{k} \leq s} B_{\gamma, s}^{\alpha} \cdot \Delta W_{k}^{\gamma}+B_{\gamma, t_{\ell}}^{\alpha}\left(W_{s}^{\gamma}-W_{t_{k}}^{\gamma}\right)\right) d W_{s}^{\prime \beta} \\
& =\sum_{h \leq k<\ell} B_{\gamma, t_{h}}^{\alpha} B_{\delta, t_{k}}^{\beta} \Delta W_{h}^{\gamma} \Delta W_{k}^{\delta}+\sum_{k \leq \ell} B_{\gamma, t_{k}}^{\alpha} B_{\delta, t_{k}}^{\beta} \Delta W_{k}^{\gamma \delta}=Z_{2, t_{\ell}}^{\prime \alpha, \beta} .
\end{aligned}
$$

Thanks to Theorem 6.4 we can introduce the concept of weak symmetry of a Milstein type discretization scheme. We can look at the solution $\bar{X}_{\ell}$ of the Milstein scheme (6.11) 
as an iterated random map defined by the geometrical $\operatorname{SDE} F(x, z)=F(x, \Delta t, \Delta W, \Delta \mathrm{W})$, where $F$ has the form

$F^{i}(x, \Delta t, \Delta W, \Delta \mathrm{W})=x^{i}+\mu^{i}(x) \Delta t_{n}+\sum_{\alpha=1}^{k} \sigma_{\alpha}^{i}(x) \Delta W^{\alpha}+\frac{1}{2} \sum_{j=1}^{n} \sum_{\alpha, \beta=1}^{k} \sigma_{\alpha}^{j}(x) \partial_{j}\left(\sigma_{\beta}^{i}\right)(x) \Delta \mathbb{W}^{\alpha, \beta}$,

and driven by the semimartingale $Z$ on the Lie group $N$ with its natural composition described above.

The weak stochastic transformation $T=(\Phi(x), B(x))$ acts on the solution to the Milstein discretization scheme in the following way $\left(X^{\prime N}, Z^{\prime}\right)=P_{T}\left(X^{N}, Z\right)$, that is

$$
\begin{aligned}
\bar{X}_{t_{\ell}}^{\prime N} & =\Phi\left(\bar{X}_{t_{\ell}}^{N}\right) \\
\Delta Z_{1, t_{\ell}}^{\prime \alpha} & =B_{\beta}^{\alpha}\left(\bar{X}_{t_{\ell-1}}^{N}\right) \Delta Z_{1, t_{\ell}}^{\beta}=B_{\beta}^{\alpha}\left(\bar{X}_{t_{\ell-1}}^{N}\right) \Delta W_{\ell}^{\beta} \\
\Delta Z_{2, t_{\ell}}^{\prime \alpha \beta} & =B_{\gamma}^{\alpha}\left(\bar{X}_{t_{\ell-1}}^{N}\right) B_{\delta}^{\beta}\left(\bar{X}_{t_{\ell-1}}^{N}\right) \Delta Z_{2, t_{\ell}}^{\gamma \delta}=B_{\gamma}^{\alpha}\left(\bar{X}_{t_{\ell-1}}^{N}\right) B_{\delta}^{\beta}\left(\bar{X}_{t_{\ell-1}}^{N}\right) \Delta \mathrm{W}_{\ell}^{\gamma \delta} \\
\Delta Z_{t_{\ell}}^{0} & =\Delta t_{\ell},
\end{aligned}
$$

is a symmetry of the discretization scheme if $\left(X^{\prime}, Z^{\prime}\right)$ is also a solution to the discretization scheme $F$. Using Theorem 4.8, a sufficient condition for having such a symmetry is that

$$
\begin{aligned}
& \Phi\left(F\left(\Phi^{-1}(x), \Delta t,\left(B \circ \Phi^{-1}\right)^{-1}(x) \cdot \Delta W,\left(B \circ \Phi^{-1}\right)^{-1}(x) \otimes\left(B \circ \Phi^{-1}\right)^{-1}(x) \cdot \Delta \mathbb{W}\right)\right. \\
& \quad=F(x, \Delta t, \Delta W, \Delta \mathbb{W}) .
\end{aligned}
$$

For a given infinitesimal stochastic transformation $(Y, C)$ the determining equations (using equation (4.9)) read

$$
\begin{gathered}
\left.Y^{j}(x) \partial_{x^{j}}\left(F^{i}\right)(x, \Delta t, \Delta W, \Delta \mathbb{W})-F^{j}(x, \Delta t, \Delta W, \Delta \mathbb{W}) \partial_{x^{j}}\left(Y^{i}\right)(x)\right)= \\
=-C_{\beta}^{\alpha}(x) \Delta W^{\beta} \partial_{\Delta W^{\alpha}}\left(F^{i}\right)(x, \Delta t, \Delta W, \Delta \mathrm{W})-C_{\beta}^{\alpha}(x) \Delta \mathbb{W}^{\beta \gamma} \partial_{W^{\alpha \gamma}}\left(F^{i}\right)(x, \Delta t, \Delta W, \Delta \mathbb{W})+ \\
-C_{\beta}^{\alpha}(x) \Delta \mathbb{W}^{\beta \gamma} \partial_{W^{\gamma \alpha}}\left(F^{i}\right)(x, \Delta t, \Delta W, \Delta \mathbb{W}) .
\end{gathered}
$$

We have the following application of Theorem 6.3 for the Milstein case.

Theorem 6.5. Suppose that $(Y, C, 0)$ is a quasi strong symmetry of the $\operatorname{SDE}(\mu, \sigma), Y^{i}$ is linear in $x^{i}$ and $C$ is a constant matrix. Then $(Y, C)$ is a weak symmetry of the Milstein discretization scheme for $(\mu, \sigma)$.

Proof. From Theorem 6.3, equation (6.13) and under the hypothesis of the present theorem, we have that $(Y, C)$ is a symmetry of the Milstein discretization if and only if

$$
\partial_{x^{j}}\left(Y^{k}\right) \sigma_{\alpha}^{i} \partial_{x^{i}}\left(\sigma_{\beta}^{j}\right) \Delta \mathbb{W}^{\alpha \beta}=Y^{j} \partial_{x^{j}}\left(\sigma_{\alpha}^{i} \partial_{x^{i}}\left(\sigma_{\beta}^{k}\right)\right) \Delta \mathbb{W}^{\alpha \beta}+\sigma_{\alpha}^{i} \partial_{x^{i}}\left(\sigma_{\beta}^{j}\right)\left(C_{\gamma}^{\alpha} \Delta \mathbb{W}^{\gamma \beta}+C_{\delta}^{\beta} \Delta \mathbb{W}^{\alpha \delta}\right) .
$$

Furthermore $(Y, C, 0)$ is a quasi-strong symmetry of the $\operatorname{SDE}(\mu, \sigma)$ if and only if

$$
-Y^{i} \partial_{x^{i}}\left(\sigma_{\alpha}^{j}\right)+\sigma_{\alpha}^{i} \partial_{x^{i}}\left(Y^{j}\right)=C_{\alpha}^{\beta} \sigma_{\beta}^{j}
$$

If we derive equation (6.15) with respect to $\sigma_{\gamma}^{i} \partial_{x^{i}}$ we obtain

$$
\begin{gathered}
-\sigma_{\alpha}^{i} \partial_{x^{i}}\left(Y^{j}\right) \partial_{x^{j}}\left(\sigma_{\beta}^{k}\right)-Y^{j} \sigma_{\alpha}^{i} \partial_{x^{i} x^{j}}\left(\sigma_{\beta}^{k}\right)+\sigma_{\alpha}^{i} \partial_{x^{i}}\left(\sigma_{\beta}^{j}\right) \partial_{x^{j}}\left(Y^{k}\right)+\sigma_{\alpha}^{i} \sigma_{\beta}^{j} \partial_{x^{i} x^{j}}\left(Y^{k}\right)= \\
=\sigma_{\alpha}^{i} \partial_{x^{i}}\left(C_{\beta}^{\gamma}\right) \sigma_{\gamma}^{k}+\sigma_{\alpha}^{i} \partial_{x^{i}}\left(\sigma_{\gamma}^{k}\right) C_{\beta}^{\gamma} .
\end{gathered}
$$

Using the fact that $Y^{i}$ is almost linear in $x^{i}$, and so $\partial_{x^{i} x^{j}}\left(Y^{k}\right)=0$, the fact that $C_{\beta}^{\alpha}$ is constant, and so $\partial_{x^{i}}\left(C_{\beta}^{\gamma}\right)=0$, and replacing equation (6.15) in equation (6.16) we obtain the relation (6.14) which means that $(Y, C)$ is a weak symmetry of the Milstein discretization scheme. 
Remark 6.6. There is an important difference between Theorem 6.3 and Theorem 6.5. Indeed Theorem 6.3 gives a necessary and sufficient condition in order that a quasi-strong symmetry $(Y, C, 0)$ of the $\operatorname{SDE}(\mu, \sigma)$ is a weak symmetry $(Y, C)$ of the Euler discretization scheme, while Theorem 6.5 gives only a sufficient condition. Furthermore the hypotheses of Theorem 6.5 request that $C_{\beta}^{\alpha}$ is a constant, while $C_{\beta}^{\alpha}$ can be any function in Theorem 6.3.

This last fact can be explained in the following way: the gauge transformation $\Xi_{B}$ transforms the process $Z=\left(Z^{0}, Z_{1}^{\alpha}, Z_{2}^{\alpha \beta}\right)$ using an Euler approximation of the usual random rotation and not a Milstein one. The two approximations of the random rotation coincide only when the rotations $B_{\beta}^{\alpha}$ (or the generator $C_{\beta}^{\alpha}$ of the rotations) are constants. Finally it is important to note that one cannot use the Milstein approximation for transforming the semimartingales $Z$, because otherwise the transformation would not preserve the law of the semimartingale $Z$.

\section{References}

[1] Sergio Albeverio, Francesco C. De Vecchi, Paola Morando, and Stefania Ugolini. Random transformations and invariance of semimartingales on Lie groups. arXiv preprint arXiv:1812.11066, 2018.

[2] Sergio Albeverio and Maria Gordina. Lévy processes and their subordination in matrix Lie groups. Bull. Sci. Math., 131(8):738-760, 2007. MR-2372464

[3] Greg W. Anderson, Alice Guionnet, and Ofer Zeitouni. An introduction to random matrices, volume 118 of Cambridge Studies in Advanced Mathematics. Cambridge University Press, Cambridge, 2010. MR-2760897

[4] David Applebaum. Lévy processes and stochastic calculus, volume 93 of Cambridge Studies in Advanced Mathematics. Cambridge University Press, Cambridge, 2004. MR-2072890

[5] David Applebaum and Serge Cohen. Stochastic parallel transport along Lévy flows of diffeomorphisms. J. Math. Anal. Appl., 207(2):496-505, 1997. MR-1438926

[6] Ludwig Arnold. Random dynamical systems. Springer Monographs in Mathematics. SpringerVerlag, Berlin, 1998. MR-1723992

[7] Martine Babillot, Philippe Bougerol, and Laure Elie. The random difference equation $X_{n}=$ $A_{n} X_{n-1}+B_{n}$ in the critical case. Ann. Probab., 25(1):478-493, 1997. MR-1428518

[8] Alan Bain and Dan Crisan. Fundamentals of stochastic filtering, volume 60 of Stochastic Modelling and Applied Probability. Springer, New York, 2009. MR-2454694

[9] Klaus Bichteler. Stochastic integration with jumps, volume 89 of Encyclopedia of Mathematics and its Applications. Cambridge University Press, Cambridge, 2002. MR-1906715

[10] George W. Bluman and Sukeyuki Kumei. Symmetries and differential equations, volume 81 of Applied Mathematical Sciences. Springer-Verlag, New York, 1989. MR-1006433

[11] Peter J. Brockwell and Richard A. Davis. Time series: theory and methods. Springer Series in Statistics. Springer-Verlag, New York, second edition, 1991. MR-1093459

[12] Rutwig Campoamor-Stursberg, Miguel A. Rodríguez, and Pavel Winternitz. Symmetry preserving discretization of ordinary differential equations. Large symmetry groups and higher order equations. J. Phys. A, 49(3):035201, 21, 2016. MR-3436668

[13] S. Cohen. Géométrie différentielle stochastique avec sauts. I. Stochastics Stochastics Rep., 56(3-4):179-203, 1996. MR-1396760

[14] S. Cohen. Géométrie différentielle stochastique avec sauts. II. Discrétisation et applications des EDS avec sauts. Stochastics Stochastics Rep., 56(3-4):205-225, 1996. MR-1396761

[15] M. Cohen de Lara. A note on the symmetry group and perturbation algebra of a parabolic partial differential equation. J. Math. Phys., 32(6):1445-1449, 1991. MR-1109191

[16] M. Cohen de Lara. Finite-dimensional filters. I. The Wei-Norman technique. SIAM J. Control Optim., 35(3):980-1001, 1997. MR-1444346

[17] M. Cohen de Lara. Finite-dimensional filters. II. Invariance group techniques. SIAM J. Control Optim., 35(3):1002-1029, 1997. MR-1444347 
[18] Mark Craddock and Kelly A. Lennox. Lie group symmetries as integral transforms of fundamental solutions. J. Differential Equations, 232(2):652-674, 2007. MR-2286394

[19] Mark Craddock and Kelly A. Lennox. Lie symmetry methods for multi-dimensional parabolic PDEs and diffusions. J. Differential Equations, 252(1):56-90, 2012. MR-2852198

[20] Ana Bela Cruzeiro, Darryl D. Holm, and Tudor S. Ratiu. Momentum maps and stochastic clebsch action principles. arXiv preprint arXiv:1604.04554, 2016. MR-3767710

[21] Christa Cuchiero, Damir Filipović, Eberhard Mayerhofer, and Josef Teichmann. Affine processes on positive semidefinite matrices. Ann. Appl. Probab., 21(2):397-463, 2011. MR2807963

[22] Francesco C. De Vecchi. Lie symmetry analysis and geometrical methods for finite and infinite dimensional stochastic differential equations. PhD thesis, Università degli Studi di Milano, 2018.

[23] Francesco C. De Vecchi and Paola Morando. The geometry of differential constraints for a class of evolution PDEs. arXiv preprint arXiv:1607.08014, 2016.

[24] Francesco C. De Vecchi, Paola Morando, and Stefania Ugolini. Reduction and reconstruction of stochastic differential equations via symmetries. J. Math. Phys., 57(12):123508, 22, 2016. MR-3590182

[25] Francesco C. De Vecchi, Paola Morando, and Stefania Ugolini. Symmetries of stochastic differential equations: A geometric approach. J. Math. Phys., 57(6):063504, 17, 2016. MR3510315

[26] Francesco C. De Vecchi, Paola Morando, and Stefania Ugolini. A note on symmetries of diffusions within a martingale problem approach. Stochastics and Dynamics, 18(6):1950011, 21, 2018. MR-3932677

[27] Francesco C. De Vecchi, Paola Morando, and Stefania Ugolini. Symmetries of stochastic differential equations using Girsanov transformations. Journal of Physics A: Mathematical and Theoretical, 2020. MR-3510315

[28] Francesco C. De Vecchi, Andrea Romano, and Stefania Ugolini. A symmetry-adapted numerical scheme for SDEs. Journal of Geometric Mechanics, 11:325-359, 2019. MR-4026012

[29] Persi Diaconis and David Freedman. Iterated random functions. SIAM Rev., 41(1):45-76, 1999. MR-1669737

[30] Vladimir Dorodnitsyn. Applications of Lie groups to difference equations, volume 8 of Differential and Integral Equations and Their Applications. CRC Press, Boca Raton, FL, 2011. MR-2759751

[31] D. Duffie, D. Filipović, and W. Schachermayer. Affine processes and applications in finance. Ann. Appl. Probab., 13(3):984-1053, 2003. MR-1994043

[32] K. D. Elworthy, Y. Le Jan, and Xue-Mei Li. On the geometry of diffusion operators and stochastic flows, volume 1720 of Lecture Notes in Mathematics. Springer-Verlag, Berlin, 1999. MR-1735806

[33] K. David Elworthy, Yves Le Jan, and Xue-Mei Li. The geometry of filtering. Frontiers in Mathematics. Birkhäuser Verlag, Basel, 2010. MR-2731662

[34] Giuseppe Gaeta. Symmetry of stochastic non-variational differential equations. Phys. Rep., 686:1-62, 2017. MR-3670079

[35] Joseph Glover and Joanna Mitro. Symmetries and functions of Markov processes. Ann. Probab., 18(2):655-668, 1990. MR-1055426

[36] Gilbert Agnew Hunt. Semi-groups of measures on Lie groups. Transactions of the American Mathematical Society, 81(2):264-293, 1956. MR-0079232

[37] Jean Jacod. Calcul stochastique et problèmes de martingales, volume 714 of Lecture Notes in Mathematics. Springer, Berlin, 1979. MR-0542115

[38] Jean Jacod and Albert N. Shiryaev. Limit theorems for stochastic processes, volume 288 of Grundlehren der Mathematischen Wissenschaften [Fundamental Principles of Mathematical Sciences]. Springer-Verlag, Berlin, second edition, 2003. MR-1943877

[39] Olav Kallenberg. Probabilistic symmetries and invariance principles. Probability and its Applications (New York). Springer, New York, 2005. MR-2161313 
[40] Harry Kesten. Random difference equations and renewal theory for products of random matrices. Acta Math., 131:207-248, 1973. MR-0440724

[41] Peter E. Kloeden and Eckhard Platen. Numerical solution of stochastic differential equations, volume 23 of Applications of Mathematics (New York). Springer-Verlag, Berlin, 1992. MR1214374

[42] Hiroshi Kunita. Stochastic differential equations based on Lévy processes and stochastic flows of diffeomorphisms. In Real and stochastic analysis, Trends Math., pages 305-373. Birkhäuser Boston, Boston, MA, 2004. MR-2090755

[43] Thomas G. Kurtz, Étienne Pardoux, and Philip Protter. Stratonovich stochastic differential equations driven by general semimartingales. Ann. Inst. H. Poincaré Probab. Statist., 31(2):351-377, 1995. MR-1324812

[44] Joan-Andreu Lázaro-Camí and Juan-Pablo Ortega. Reduction, reconstruction, and skewproduct decomposition of symmetric stochastic differential equations. Stoch. Dyn., 9(1):1-46, 2009. MR-2502472

[45] Decio Levi, Peter Olver, Zora Thomova, and Pavel Winternitz, editors. Symmetries and integrability of difference equations, volume 381 of London Mathematical Society Lecture Note Series. Cambridge University Press, Cambridge, 2011. Lectures from the Summer School (Séminaire de Máthematiques Supérieures) held at the Université de Montréal, Montréal, QC, June 8-21, 2008. MR-2868097

[46] Decio Levi and Pavel Winternitz. Continuous symmetries of difference equations. J. Phys. A, 39(2):R1-R63, 2006. MR-2198959

[47] Ming Liao. Lévy processes in Lie groups, volume 162 of Cambridge Tracts in Mathematics. Cambridge University Press, Cambridge, 2004. MR-2060091

[48] Ming Liao. Markov processes invariant under a Lie group action. Stochastic Process. Appl., 119(4):1357-1367, 2009. MR-2508577

[49] Steven I. Marcus. Modeling and approximation of stochastic differential equations driven by semimartingales. Stochastics, 4(3):223-245, 1980/81. MR-0605630

[50] Madan Lal Mehta. Random matrices, volume 142 of Pure and Applied Mathematics (Amsterdam). Elsevier/Academic Press, Amsterdam, third edition, 2004. MR-2129906

[51] Peter J. Olver. Applications of Lie groups to differential equations, volume 107 of Graduate Texts in Mathematics. Springer-Verlag, New York, second edition, 1993. MR-1240056

[52] Philip Protter. Stochastic integration and differential equations, volume 21 of Applications of Mathematics (New York). Springer-Verlag, Berlin, 1990. A new approach. MR-1037262

[53] Sebastian J. Schreiber. Persistence for stochastic difference equations: a mini-review. J. Difference Equ. Appl., 18(8):1381-1403, 2012. MR-2956051

[54] Ė. B. Vinberg, editor. Lie groups and Lie algebras, III, volume 41 of Encyclopaedia of Mathematical Sciences. Springer-Verlag, Berlin, 1994.

[55] Jean-Claude Zambrini. The research program of stochastic deformation (with a view toward geometric mechanics). In Stochastic analysis: a series of lectures, volume 68 of Progr. Probab., pages 359-393. Birkhäuser/Springer, Basel, 2015. MR-3558132

Acknowledgments. The first author would like to thank the Department of Mathematics, Università degli Studi di Milano for the warm hospitality. This research was supported by IAM and HCM (University of Bonn) and Gruppo Nazionale Fisica Matematica (GNFMINdAM). The second author is supported by the German Research Foundation (DFG) via CRC 1060. 